\title{
Entendiendo los Cambios en la Segregación Escolar. Un Análisis en Base a Microdescomposiciones
}

\author{
Understanding the Changes in School Segregation. An Analysis \\ Based on Microdecompositions
}

\author{
Lucía Ramírez Leira ${ }^{1,2}$ * \\ Emmanuel Vazquez ${ }^{1}$ \\ ${ }^{1}$ CEDLAS-IIE-FCE-Universidad Nacional de La Plata, Argentina \\ ${ }^{2}$ IECON, Universidad de la República, Uruguay
}

\begin{abstract}
La separación de estudiantes de diferentes entornos socioeconómicos entre escuelas públicas y privadas ha aumentado en numerosos países en los últimos años. Este trabajo propone una metodología para descomponer los cambios en la segregación escolar en el tiempo y cuantificar la importancia relativa de diferentes factores que dan cuenta de esos cambios. Para ilustrar su utilidad, el estudio provee una aplicación empírica al caso uruguayo, uno de los países de América Latina que mayor incremento de la segregación escolar entre escuelas públicas y privadas ha experimentado en los últimos años. El trabajo encuentra que los cambios en las preferencias por asistir a la escuela dan cuenta de una fracción muy pequeña del aumento de la segregación en Uruguay entre 1992 y 2017. En cambio, las preferencias por asistir a un establecimiento del tipo privado tuvieron un rol importante en el cambio observado en la segregación, particularmente en secundaria, mientras que los cambios en la restricción presupuestaria, captados vía modificaciones en los ingresos, explican la mayor parte del cambio observado en la segregación durante el período. Pese a las limitaciones de la metodología que se discuten en el trabajo, se propone su utilización para enriquecer el análisis de los determinantes de la segregación escolar y ayudar a desentrañar el aporte relativo de diferentes teorías que pueden dar cuenta de los cambios observados.
\end{abstract}

Descriptores: Segregación; Elección de escuela; Educación; Microdescomposiciones Uruguay.

\begin{abstract}
The separation of students from different socioeconomic backgrounds between public and private schools has increased in many countries during the past few years. This article proposes a methodology to decompose changes in school segregation over time and quantify the relative importance of the different factors that may account for these changes. To illustrate its usefulness, the study provides an empirical application to the Uruguayan case, one of the Latin American countries that has experienced the greatest increase in school segregation between public and private schools in recent years. The article finds that changes in preferences for attending school account for a very small fraction of the increase in segregation in Uruguay between 1992 and 2017. In contrast, preferences for attending a private type institution played an important role in the observed change in segregation, particularly in secondary education, while changes in the budget constraint, captured via changes in income, explain most of the observed change in segregation during the period. Despite the limitations of the methodology that are discussed in the article, its use is proposed to enrich the analysis of the determinants of school segregation and help to unravel the relative contribution of different theories that are able to account for the observed changes.
\end{abstract}

Keywords: Segregation; School choice; Education; Microdecompositions; Uruguay.

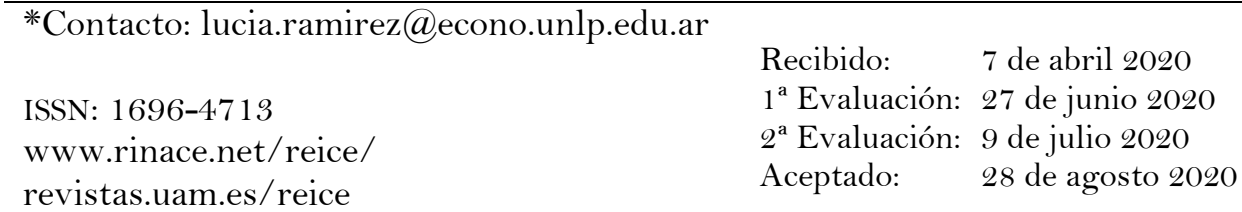




\section{Introducción}

Durante mucho tiempo, la escuela pública constituyó en varios países del mundo un espacio de interacción clave para aprender a convivir con personas provenientes de estratos sociales diferentes e igualar oportunidades. A la escuela pública asistían personas de distintas clases sociales; pobres y ricos se mezclaban en las aulas y recibían una educación semejante. En las últimas décadas, sin embargo, la mayoría de estos países ha presenciado un fuerte crecimiento de la matrícula en escuelas privadas, impulsado por la migración selectiva de los grupos no carenciados desde la escuela pública a la privada. Este fenómeno ha sido particularmente importante en América Latina (Arcidiácono et al., 2014; Murillo, 2016; Murillo y Martínez-Garrido, 2017), pero no exclusivo de ella (véase, por ejemplo, Murillo, Belavi y Pinilla, 2018 para España) y ha dado forma al fenómeno de segregación escolar, por el cual estudiantes de distinto nivel socioeconómico ya no interactúan de igual manera en las aulas, porque asisten a diferentes tipos de establecimientos.

La segregación escolar, entendida aquí como la separación de alumnos de distinto nivel socioeconómico entre centros educativos públicos y privados $^{1}$, tiene profundas consecuencias sobre la sociedad: contribuye a la generación de fenómenos de exclusión y desintegración en otros ámbitos (Gasparini et al., 2011); da lugar a la generación de desigualdades persistentes y trampas de pobreza (Durlauf, 2006); estrecha las posibilidades y contactos de los grupos más vulnerables, afectando además su probabilidad de exposición a códigos, mensajes y conductas necesarias para una movilidad social ascendente (Valenzuela, 2008). Finalmente, cuando la segregación es elevada puede también amenazar la cohesión social existente (Jenkins, Micklewright y Schnepf, 2008; Wilson, 1987). En efecto, cuando distintas clases sociales no se "mezclan" en espacios comunes, no pueden comunicarse, conocerse, ni aprender las necesidades y motivaciones del otro, lo que vuelve particularmente difícil entenderlo, favoreciendo el prejuicio y la estigmatización, e incrementando las posibilidades de un mayor conflicto social en el futuro.

Entender los determinantes de la segregación escolar ha sido todo un desafío para la literatura. Múltiples explicaciones desde diferentes ramas de la economía y la sociología han sido ofrecidas. La economía típicamente le ha asignado un rol importante a las preferencias y a la restricción presupuestaria de los individuos. En efecto, si las preferencias por los centros educativos o su composición socioeconómica son heterogéneas, la segregación ocurre con un resultado de equilibrio fácilmente (como en modelos al estilo Schelling, 1971 o Card, Mas y Rothstein, 2008). Si los individuos tienen en cambio las mismas preferencias, la segregación se obtiene como resultado a través del conflicto sobre recursos escasos, surgiendo a través de mecanismos explícitos o implícitos de racionamiento del acceso a las escuelas (como en los modelos de equilibrio parcial de Becker y Murphy, 2003 y de equilibrio general de Nechyba, 2006, o Epple y Romano, 1998, donde las diferencias de precios e ingresos son claves).

\footnotetext{
${ }^{1}$ El presente trabajo se centra en la segregación entre el agregado de escuelas públicas y privadas, que explica un porcentaje importante de la segregación total en América Latina (Vazquez, 2012, 2016).
} 
Entender los cambios que han ocurrido recientemente en la segregación escolar requiere cuantificar la importancia relativa que han tenido los distintos determinantes de la decisión de asistencia a un establecimiento educativo y de la selección de un centro educativo del tipo público o privado. Aunque resulta imposible estimar un modelo completo que capte todos los determinantes de este cambio, este trabajo propone una metodología para cuantificar la importancia relativa que tienen los cambios en las preferencias y en la restricción presupuestaria de los individuos sobre la segregación escolar. Para ello, propone una microdescomposición de los cambios en la segregación y realiza una aplicación empírica al caso de Uruguay. Este país constituye un caso de estudio interesante, ya que, junto con Argentina y Perú, ha sido el país que ha experimentado un mayor aumento en la segregación entre escuelas públicas y privadas entre las décadas de 1990 y 2010 en América Latina (Arcidiácono et al., 2014)².

El estudio encuentra que los cambios en las preferencias por asistir a la escuela dan cuenta de una fracción muy pequeña del aumento que ha experimentado la segregación en Uruguay entre 1992 y 2017. En cambio, las preferencias por asistir a un establecimiento del tipo privado tuvieron un rol importante en el cambio observado en la segregación, particularmente en secundaria, lo cual es consistente con el hecho de que las brechas de calidad que se perciben entre escuelas públicas y privadas han aumentado en el período considerado. Asimismo, los cambios en la restricción presupuestaria, captados vía modificaciones en los ingresos, explican la mayor parte del cambio observado en la segregación durante el período, lo que sugiere que el ingreso ha sido el principal determinante de la evolución en la segregación escolar en el caso uruguayo.

El resto del trabajo está organizado de la siguiente forma. La Sección 2 desarrolla la metodología. La Sección 3 describe los datos utilizados para ilustrar la metodología con una aplicación empírica, proveyendo el contexto general del caso analizado. La Sección 4 muestra los resultados de las microdescomposiciones del cambio en la segregación escolar aplicados a este caso, mientras que la Sección 5 concluye con algunos comentarios finales.

\section{Metodología}

\subsection{Microdescomposición propuesta}

La metodología de microdescomposiciones propuesta en este trabajo para explicar los cambios en la segregación educativa constituye una extensión de la metodología diseñada por Bourguignon, Ferreira y Lustig (2005), que permite medir la importancia que pueden haber tenido distintos factores en el cambio de la segregación entre los momentos $t$ y $t^{\prime}$. Si bien la metodología se diseñó originalmente para ser aplicada a temas distributivos, es posible utilizarla en este caso para determinar cuánto del cambio en la segregación en un período analizado está relacionado con cambios en características observables e inobservables de los asistentes a la educación pública y privada; y cuánto con cambios en los parámetros que gobiernan sus decisiones de asistencia.

${ }^{2}$ De acuerdo con el mencionado estudio, Uruguay fue el país que experimentó el mayor incremento de la segregación entre escuelas públicas y privadas medida por el índice de Brecha por centiles entre 1992 y 2011, y el tercer mayor aumento de acuerdo al índice de Disimilitud, detrás de Perú y Argentina. 
La idea básica consiste en comparar las decisiones de asistencia a centro educativos públicos/privados en el momento $t$, con decisiones contrafactuales simuladas. Estas decisiones contrafactuales surgen de simular cómo serían las decisiones de asistencia y elección público/privado en el momento $t$ si algunos de los factores fueran los del momento $t^{\prime}$.

A continuación, se presenta analíticamente la metodología propuesta, que sigue de cerca a la empleada en Gasparini (2006) para descomponer los cambios en la incidencia distributiva del gasto público. Se supone una población de tamaño $\mathrm{N}$, donde cada uno de sus integrantes se indexa por $i=1, \ldots, N$. Cada individuo se identifica por un vector de características observables $X_{i}$ y no observables $U_{i}$.

Dentro de la población total, los individuos en edad escolar pueden decidir asistir o no a un centro educativo. Condicional a su decisión de asistencia, eligen posteriormente si concurren a un centro educativo público o privado. Sea $d_{i t}$ una variable binaria que vale 1 si el individuo $i$ está en edad escolar y asiste a una escuela pública en el momento $t$. Entonces, podemos escribir

$$
d_{i t}=c_{i t} * a_{i t} * p_{i t}
$$

donde $c$ vale 1 si el individuo se encuentra en edad escolar y 0 en caso contrario; $a$ es igual a 1 si el individuo asiste a un centro educativo dado que está en edad escolar y o en caso contrario; y $p$ toma el valor 1 si el individuo, una vez que decide asistir, elige una institución pública.

Sea $\delta_{t}$ el vector de parámetros que determinan la regla de acceso (en este caso, tener edad escolar) en el momento $t$. Las características que determinan si un individuo cumple o no la regla de acceso son observables, por lo que se asume que $c$ es determinística:

$$
c_{i t}=C\left(X_{i t} ; \delta_{t}\right)
$$

Por su parte, debido a que la decisión de asistencia y de elección público/privada depende de factores observables e inobservables, $a_{i t} \mathrm{y} p_{i t}$, son consideradas variables aleatorias:

$$
\begin{aligned}
& a_{i t}=A\left(X_{i t} ; U_{i t} ; \beta_{t}\right) \\
& p_{i t}=P\left(X_{i t} ; U_{i t} ; \lambda_{t}\right)
\end{aligned}
$$

Combinando las ecuaciones anteriores, se obtiene:

$$
d_{i t}=D\left(X_{i t} ; U_{i t} ; \delta_{t} ; \beta_{t} ; \lambda_{t}\right)
$$

Por tanto, la asistencia a un centro educativo público/privado del individuo $i$ en el momento $t$ depende de características observables $X_{i t}$ que afectan tanto la asistencia educativa como la elección del tipo de centro al que asiste, de un vector de características inobservables $U_{i t}$ y un vector de parámetros $\beta_{t}$ (que determinan la asistencia) y otro $\lambda_{t}$ (que conducen la elección público/privado).

Por su parte, los indicadores normalmente utilizados para medir la segregación educativa $S_{t}$ son una combinación de la distribución de $d_{i t}$ y de algunas características $r$ del vector $X$ (por lo general, los ingresos de los individuos), por lo que pueden expresarse analíticamente como:

$$
S_{t}=F\left(\left\{X_{i t}\right\},\left\{U_{i t}\right\}, \delta_{t}, \beta_{t}, \lambda_{t}\right)
$$




$$
S_{t^{\prime}}=F\left(\left\{X_{i t^{\prime}}\right\},\left\{U_{i t^{\prime}}\right\}, \delta_{t^{\prime}}, \beta_{t^{\prime}}, \lambda_{t^{\prime}}\right)
$$

La ecuación (5) define los determinantes de la asistencia a educación pública/privada del individuo $i$ en el momento $t$ y que serán objeto de la simulación. La idea detrás de esta descomposición es sustituir gradualmente los argumentos de dicha ecuación en el momento $t$ por los del momento $t^{\prime}$.

Esta sustitución gradual, permitirá descomponer los cambios observados en la segregación $\left(\Delta S=S_{t^{\prime}}-S_{t}\right)$ en tres efectos:

- Efecto participación (PA): captura la variación de la segregación educativa que surge de cambios en los parámetros que determinan la asistencia. Analíticamente, este efecto puede expresarse como:

$$
P A=F\left(\left\{X_{i t^{\prime}}\right\},\left\{U_{i t^{\prime}}\right\}, \delta_{t^{\prime}}, \beta_{t^{\prime}}, \lambda_{t^{\prime}}\right)-F\left(\left\{X_{i t^{\prime}}\right\},\left\{U_{i t^{\prime}}\right\}, \delta_{t^{\prime}}, \beta_{t}, \lambda_{t^{\prime}}\right)
$$

- Efecto provisión pública (PP): refleja la variación de la segregación educativa que surge de cambios en los parámetros que gobiernan la elección de un centro educativo público o privado. Analíticamente, este efecto puede expresarse como:

$$
P P=F\left(\left\{X_{i t^{\prime}}\right\},\left\{U_{i t^{\prime}}\right\}, \delta_{t^{\prime}}, \beta_{t}, \lambda_{t^{\prime}}\right)-F\left(\left\{X_{i t^{\prime}}\right\},\left\{U_{i t^{\prime}}\right\}, \delta_{t^{\prime}}, \beta_{t}, \lambda_{t}\right)
$$

- Efecto características (PC): representa la variación de la segregación educativa que surge de cambios en las características observables e inobservables de la población. Analíticamente, este efecto puede expresarse como:

$$
P C=F\left(\left\{X_{i t^{\prime}}\right\},\left\{U_{i t^{\prime}}\right\}, \delta_{t^{\prime}}, \beta_{t}, \lambda_{t}\right)-F\left(\left\{X_{i t}\right\},\left\{U_{i t}\right\}, \delta_{t}, \beta_{t}, \lambda_{t}\right)
$$

En tanto la regla de acceso no cambie en el período considerado $\left(\delta_{t}=\delta_{t^{\prime}}\right)$, el cambio en el indicador de segregación puede expresarse como:

$$
\Delta S=P A+P O+P C
$$

Una innovación metodológica de este trabajo consiste en cuantificar cuál es el aporte específico de ciertas características dentro del efecto características general que surge de la descomposición anterior. Para aproximarse a la importancia particular de la característica $x_{k}$, es posible simular la decisión de asistencia y elección de educación pública que el individuo $i$ hubiese obtenido en el momento $t$, al sustituir el valor de $x_{k}$ por el correspondiente en $t^{\prime}$, manteniendo el resto de los argumentos constantes. Dado que reemplazar $x_{k}$ de $t^{\prime}$ en los datos de $t$ arroja resultados diferentes que cambiar $x_{k}$ de $t$ en los datos de $t^{\prime}$, se propone calcular los escenarios contrafácticos de una y otra forma $\mathrm{y}$ computar el promedio de ambos. En base a esto, puede obtenerse una distribución de asistencia público y privada observada $\left(D_{t}\right)$ y una simulada $\left(D_{t r k^{\prime}}\right)$. El efecto marginal del cambio en la característica $\mathrm{x}_{\mathrm{k}}$ sobre la segregación se obtiene al comparar el indicador de segregación $(S)$ calculado sobre la distribución observada y la simulada de estudiantes que asisten a público y a privado según su nivel socioeconómico:

$$
S\left(D_{t}\right)-S\left(D_{t x k^{\prime}}\right)
$$

De lo anterior se desprende que previo a realizar las microdescomposiciones, es necesario estimar un modelo de asistencia y de elección público/privado. Si bien ésta última decisión puede estar afectada por diversos procesos que no siempre pueden captarse con los datos disponibles, la asistencia a un centro público o privado parece estar vinculada también a 
decisiones de las familias que, dadas sus preferencias y restricciones, toman sus decisiones respecto a asistir o no al sistema educativo público.

El problema de la elección de una escuela pública puede ser interpretado como un modelo de elección secuencial, donde la familia primero decide si el individuo asiste o no a una escuela, y en función de su decisión de asistencia, opta por un centro público o privado.

Si se supone en términos generales que cada posible elección $i$ reporta una utilidad $U_{i}$, es posible escribir la probabilidad de que un individuo decida asistir a un centro educativo público como una combinación de las probabilidades de cada opción condicional a la elección de la etapa previa:

$$
\begin{gathered}
P[\text { asiste público }]=P[\text { asiste }] \times P[\text { público } \mid \text { asiste }]=P\left[U_{\text {asiste }} \geq\right. \\
\left.U_{\text {noasiste }}\right] \times P\left[U_{\text {público }} \geq U_{\text {privado }} \mid \text { asiste }=1\right]
\end{gathered}
$$

Si se supone ese proceso decisorio, lo anterior podría resultar en un modelo probit condicional o un modelo logit anidado. Debido a que para el análisis de microdescomposiciones se requiere recuperar los parámetros de cada una de las etapas decisorias por separado, se propone estimar secuencialmente un modelo no lineal de probabilidad para cada una de las decisiones.

$$
\begin{gathered}
P[\text { asistencia }=1]=F(X \beta) \\
P[\text { público }=1 \mid \text { asistencia }=1]=F(X \lambda)
\end{gathered}
$$

donde $F($.$) es la función de distribución normal.$

Las estimaciones de dichas probabilidades permiten obtener una probabilidad predicha $\hat{a}_{i t}$ de asistir a la escuela y una probabilidad predicha $\hat{p}_{i t}$ de elegir una institución pública para el individuo $i$ en el momento $t$. En base a estas probabilidades predichas utilizando los datos observados, se obtiene un indicador estimado para el momento $t$.

Posteriormente, se modifica alguno de los argumentos de la ecuación (5) por los del momento $t^{\prime}$ para estimar nuevas probabilidades de asistencia y elección público/privado. Finalmente, se compara el valor del indicador estimado utilizando la distribución real de asistentes de distinto nivel socioeconómico a centros públicos y privados con el valor del índice estimado que se obtiene considerando la distribución simulada.

La metodología propuesta difiere de otros aportes relevantes tales como el de Jaume (2013) en tanto permite cuantificar cuál es el aporte específico de ciertas características dentro del efecto características general. De esta manera, si se incorporan elementos de la restricción presupuestaria de los individuos (tales como el ingreso) entre las características individuales determinantes de la elección de escuela, es posible cuantificar en cierto modo cuánto de un aumento de la segregación puede atribuirse a un mayor deseo por asistir a un tipo de establecimiento y cuánto a una mayor posibilidad de asistir al mismo. Al mismo tiempo, al proponer modelar la decisión de asistencia de manera secuencial, permite estimar un efecto participación y un efecto provisión pública por separado, lo cual tampoco reconoce antecedentes en la literatura de segregación escolar.

A los efectos de medir el fenómeno que es objeto de este estudio, en la aplicación empírica se utilizará el índice de Disimilitud (D) propuesto por Duncan y Duncan (1955), que se define como: 


$$
D=\frac{1}{2} \sum_{i=1}^{k}\left|\frac{x_{1 i}}{X_{1}}-\frac{x_{2 i}}{X_{2}}\right|
$$

donde $i$ indexa en este caso el tipo de establecimiento escolar (público o privado), $x_{1 i}$ representa el número de alumnos vulnerables (de bajo nivel socioeconómico) en el tipo de establecimiento $i, X_{l}$ es el total de alumnos vulnerables, $x_{2 i}$ es el número de estudiantes no vulnerables en el establecimiento $i$ y $X_{2}$ es el total de estudiantes no vulnerables. Este índice refleja la proporción de estudiantes del grupo minoritario, en este caso los individuos vulnerables, que deberían cambiar de tipo de centro educativo para que exista una distribución homogénea de los mismos entre los dos tipos de establecimientos educativos. El indicador toma valores entre o y 1, donde o indica ausencia de segregación y 1 presencia de segregación extrema.

\subsection{Interpretación y limitaciones}

En la explicación detallada arriba, los efectos participación y provisión pública son obtenidos estimando cuán distinta hubiese sido la segregación en el momento $t^{\prime}$ si las características de los individuos (entre ellas, el ingreso) no hubiesen cambiado en el período considerado entre $t$ y $t^{\prime}$. Estos cambios pueden ser interpretados, en un sentido amplio, como un cambio en las preferencias de los individuos, que con las mismas características desean ahora una mayor participación escolar o una menor participación en el sector público.

Naturalmente, esta interpretación de los efectos parámetros PA y PP abarca no sólo cambios en las preferencias propias de los individuos (un efecto de primer orden), sino también cambios en las preferencias por la participación o el tipo de establecimiento producto de cambios en las características de las escuelas que se traducen en un mayor o menor deseo de adquirir el bien educación y asistir a un tipo de establecimiento determinado (un efecto de segundo orden, en el sentido que se deriva de cambios en las características del sistema en lugar de las características individuales).

Esta interpretación de cambios en las preferencias en sentido amplio es la que se propone para contrastar el peso de estos determinantes en oposición a las características de los individuos. Asimismo, la metodología propone la descomposición parcial del efecto características para poder cuantificar la importancia de características específicas de los individuos, tales como su ingreso, que tienen un rol fundamental en la elección de educarse y el tipo de escuela a elegir.

La metodología tiene, como es sabido, las limitaciones típicas de cualquier microdescomposición econométrica, lo que hace que los efectos estimados no sean de equilibrio general. A su vez, pese a ser una herramienta útil para cuantificar la contribución de varios factores al cambio en la segregación, la metodología no busca recuperar las relaciones conductuales o los parámetros estructurales profundos. Sin embargo, al indicar qué factores son cuantitativamente importantes y cuáles no, nos provee de una indicación de qué hipótesis particulares o teorías deben ser exploradas con más detalle (Fortin, Lemieux y Firpo, 2011).

\section{Aplicación empírica: Contexto}

A los efectos de ilustrar la metodología propuesta con un caso práctico, el presente trabajo realiza una descomposición de los cambios en la segregación experimentados por uno de 
los países de América Latina que mayor aumento ha experimentado en la separación de alumnos de distinto nivel socioeconómico entre establecimientos públicos y privados: Uruguay. El período analizado será el comprendido entre los años 1992 y 2017 y el análisis estará concentrado en los individuos entre 6 y 17 años que asisten a educación primaria o educación secundaria. Esta sección realiza una descripción del contexto de ese país en el período bajo análisis.

En Uruguay, la educación primaria y secundaria es obligatoria. La educación primaria atiende a los niños a partir de los 6 años de edad y comprende seis grados. Por su parte, la educación secundaria, en términos generales, está organizada en dos ciclos obligatorios, cada uno de tres años de duración: el ciclo básico y el ciclo superior. Ambos ciclos se ofrecen en modalidad general o en modalidad tecnológica o técnica ${ }^{3}$. Tanto en primaria como en secundaria, las personas pueden elegir entre establecimientos públicos, que son gratuitos, o establecimientos privados, que exigen el pago de una cuota mensual para poder educarse en ellos.

La población uruguaya tiene una visión más positiva sobre la educación privada que sobre la pública: la Encuesta de opinión pública sobre educación obligatoria en Uruguay 2015 (Ineed, 2017) revela que casi la mitad de la población considera que las oportunidades de aprender son mayores en la educación privada, mientras que la otra mitad considera que no hay diferencias entre ambos subsistemas. Asimismo, la población considera que la educación en general ha empeorado. Los principales motivos refieren a la cantidad y calidad docente y a los paros, las medidas sindicales y el ausentismo docente, que se dan mayormente en las instituciones públicas. La comparación de los resultados de esta encuesta con los de Latinobarómetro de 2011 muestra además que ha habido una mejora en la evaluación del sector privado por parte de la población, mientras que la valoración del sector público se mantuvo estable, lo cual sugiere que la brecha de calidad percibida entre ambos tipos de instituciones se ha ampliado en los últimos años.

Los datos muestran que el total de alumnos que asisten a la educación primaria y secundaria en Uruguay en el período 1992-2017 ha aumentado, aunque escasamente. La figura 1 permite identificar dos sub-períodos en el análisis de la evolución de la matrícula. El período 1992-2003 muestra un crecimiento del total de alumnos asistentes a educación primaria y secundaria; mientras que durante 2004-2017 se observa una matrícula estable, aunque levemente decreciente. La disminución del total de alumnos en los últimos quince años no responde a una caída en la tasa de escolarización sino a una reducción de la población en edad de asistir a la educación primaria y secundaria. En la figura 1 puede observarse también que la matrícula de la educación privada presenta un patrón inverso al de la educación pública, lo que deriva en un aumento de la participación de la educación privada durante los últimos 15 años, ya que la matrícula total se mantuvo prácticamente constante.

Para el análisis que sigue, al igual que para el cómputo de la segregación y su posterior descomposición, se utilizan datos de la Encuesta Continua de Hogares (ECH) de Uruguay, cuyos microdatos han sido publicados por el Instituto Nacional de Estadística (INE) de ese país y armonizados por el Centro de Estudios Distributivos Laborales y Sociales

\footnotetext{
${ }^{3}$ A los efectos de este trabajo, se considera educación secundaria a todos los ciclos correspondientes a la educación media, es decir, todos los ciclos que se ofrezcan luego de haber completado la educación primaria y que no sean considerados educación terciaria.
} 
(CEDLAS) y el Banco Mundial en la Base de Datos Socioeconómicos para América Latina y el Caribe (SEDLAC) ${ }^{4}$.

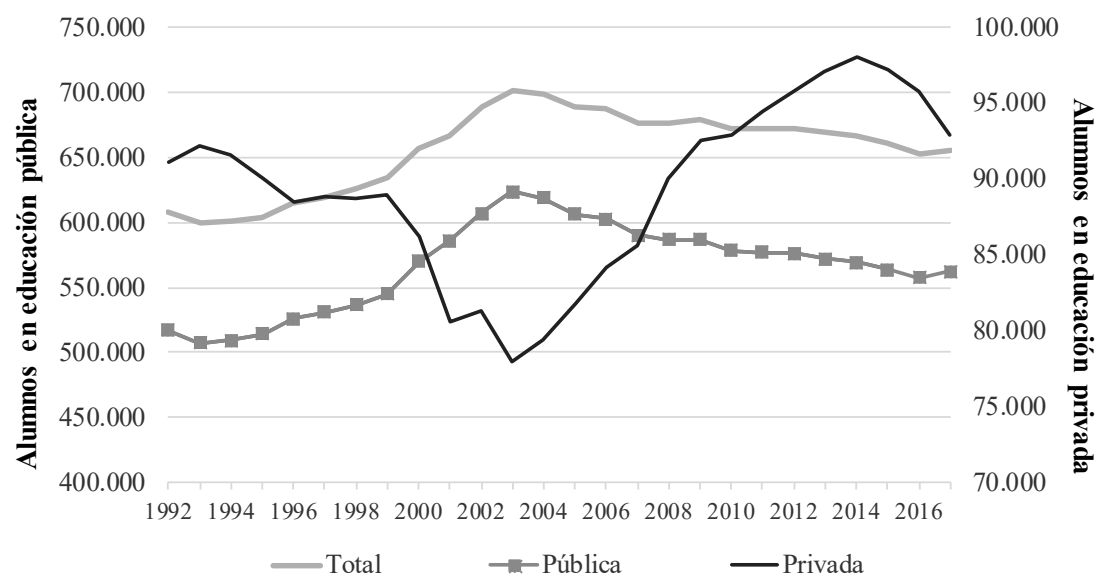

Figura 1. Matrícula según tipo de administración. 1992-2017

Fuente: Elaboración propia en base a datos del Observatorio de la educación (ANEP).

La figura 2 muestra que la tasa de escolarización ha aumentado a lo largo del período tanto para los sectores más ricos como para los más pobres. En la educación primaria se observa que la brecha entre el primer y quinto quintil (que era de aproximadamente 3 puntos porcentuales) se cierra sobre finales del período, superando el 99\% de asistencia para todos los quintiles de ingreso. Respecto a la educación secundaria, se observa un incremento diferenciado según quintil de ingreso de las tasas de escolarización, donde la asistencia de los estudiantes pobres ha crecido a mayor tasa que la asistencia de los no pobres; por lo que la brecha de asistencia entre el quintil 1 y el quintil 5 se redujo a 13 puntos porcentuales.

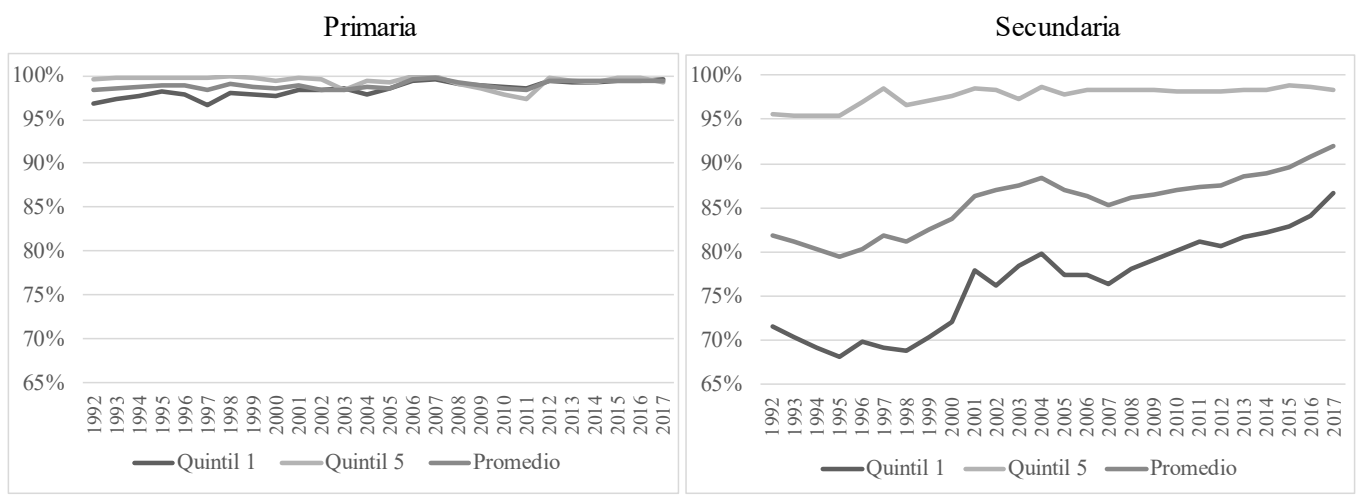

Figura 2. Tasa de escolarización de individuos en edad escolar. Total país

Nota: La tasa de escolarización representa la proporción de individuos en edad de asistir correspondiente a cada nivel que declaran asistir a un centro educativo.

Fuente: Elaboración propia en base a ECH.

\footnotetext{
${ }^{4}$ Como la ECH ha tenido cambios en la cobertura geográfica durante el período, se homogenizan las áreas empleadas en el análisis a nivel país, de manera de que en cada año los datos son representativos de las localidades de 5000 habitantes o más.
} 
La figura 3 ilustra el fenómeno de migración que se ha producido del sistema educativo público hacia el privado en los estratos medios y altos. Para el primer quintil de ingresos parece prácticamente no haberse alterado la importancia relativa de la educación pública en todo el período, posiblemente porque la educación privada exige el pago de una matrícula que no pueden afrontar. Por su parte, los quintiles del medio son los que más modifican su comportamiento, y ese comportamiento coincide con la evolución del ingreso per cápita. Entre 1992 y 2003 el ingreso de los hogares disminuyó (considerando punta a punta) y los quintiles 2 , 3 y 4 eligieron en mayor proporción la educación pública. Por su parte, entre el 2004 y el 2017 el ingreso de los hogares ha aumentado sostenidamente y se observa una migración hacia el sector privado fundamentalmente en los últimos tres quintiles ${ }^{5}$. En particular, la educación pública para el cuarto quintil disminuye 30 puntos porcentuales y para el último quintil 20 puntos porcentuales.

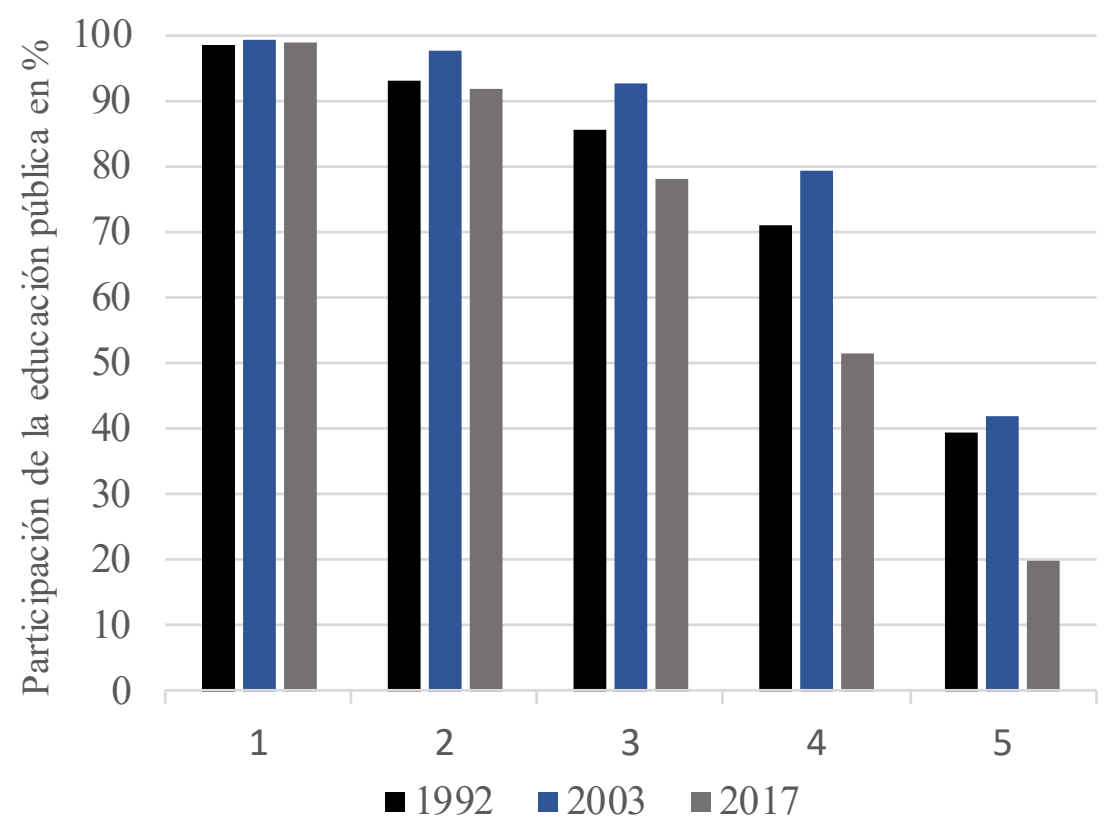

Figura 3. Participación de la educación pública según quintil de ingreso Fuente: Elaboración propia en base a ECH.

El resultado de todos estos cambios es la evolución de la segregación escolar que se muestra en la figura 4, que utiliza como definición de grupo minoritario a los individuos pertenecientes al primer quintil del ingreso per cápita familiar (también llamados pobres o vulnerables). El índice refleja que en 1992 el 21,2 \% de los alumnos pobres de todo el país debía trasladarse al sistema educativo privado para que los centros educativos de ambas modalidades sean homogéneos, mientras que para el año 2017 este valor asciende a $29,1 \%$, reflejando un aumento de $37 \%$ para el total nacional. Montevideo presenta para todos los años valores más altos, reflejando un mayor nivel de segregación, con un aumento del $30 \%$.

${ }^{5}$ En el cuadro A1 del anexo se presenta la evolución del ingreso de los hogares en Uruguay durante el período de estudio. 


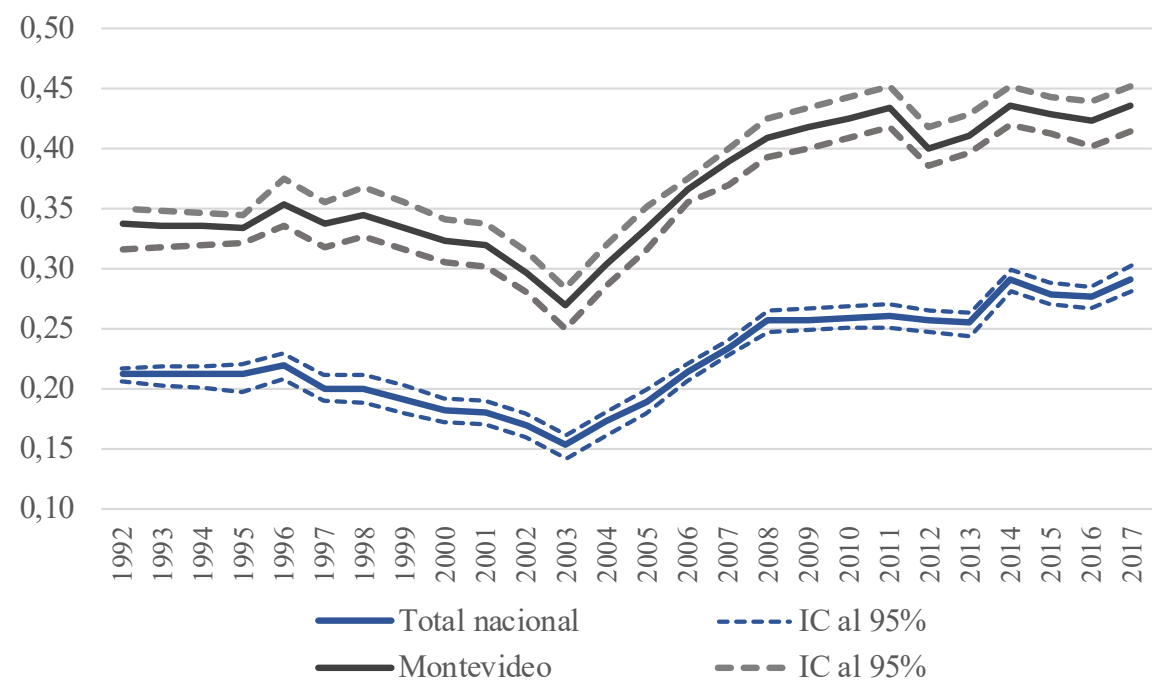

Figura 4. Índice de Disimilitud. Total educación básica. 1992-2017 Fuente: Elaboración propia en base a ECH.

El análisis separado según nivel educativo, que se muestra en la figura 5, refleja en términos generales los mismos resultados, ya que los índices de ambos niveles evolucionan de forma similar. El nivel primario parece tener mayor nivel de segregación que el nivel secundario, donde los valores de disimilitud son entre un $15 \%$ y un $30 \%$ superiores en el primero de ellos, ya sea en Montevideo o en todo el país.

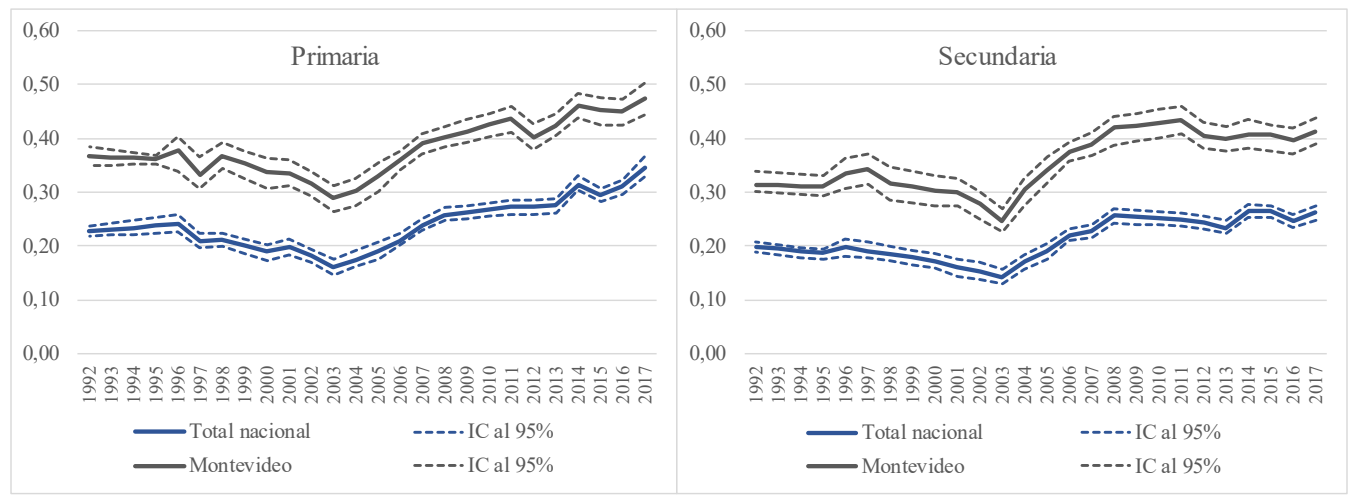

Figura 5. Índice de Disimilitud según nivel educativo. 1992-2017

Fuente: Elaboración propia en base a datos de ECH.

\section{Aplicación empírica: Resultados}

En esta sección se presentan los resultados de aplicar la metodología de microdescomposiciones propuesta para explicar los cambios en la segregación ocurridos en Uruguay a nivel nacional, considerando tres intervalos de tiempo que resultan relevantes dado el análisis del contexto de la sección anterior: el período 1992-2017, y los sub-períodos 1992-2003 y 2003-2017.

En los cuadros A3, A4 y A5 del Anexo se presentan las estimaciones de la probabilidad de asistencia y de elección de educación pública necesarias para llevar a cabo la descomposición, detallando en el cuadro A2 las variables utilizadas. Si bien existen 
algunas diferencias entre los años considerados, en términos generales se observan resultados similares a los encontrados en la literatura revisada. La probabilidad de asistencia se correlaciona positivamente con el ingreso, la educación de los padres, si el jefe de hogar se encuentra ocupado, si el jefe y el cónyuge forman parte de la población económicamente activa; mientras que se reduce cuando aumenta la edad, si el individuo es varón y si vive en una zona metropolitana. Por su parte, la probabilidad de optar por un centro educativo público aumenta cuando aumenta la edad, se reduce el ingreso, el nivel educativo de los padres es menor, el hogar es no nuclear, y el lugar de residencia no está en el área metropolitana ${ }^{6}$.

\subsection{Total Educación básica}

El cuadro 1 presenta los resultados de las microdescomposiciones para el índice de Disimilitud considerando a primaria y secundaria conjuntamente. Tal como se observa, el índice de Disimilitud aumentó o,087 en el período 1992-201777. El efecto participación allí presentado refleja que si lo único que se hubiera modificado entre 1992 y 2017 son los parámetros que gobiernan la decisión de asistencia, el índice de segregación habría caído 0,005, aunque la baja magnitud de este cambio indica que este efecto parece haber sido casi irrelevante para explicar los cambios en la segregación. Por su parte, el efecto de provisión pública contribuyó a un aumento de 0,034 del índice de Disimilitud (39\% del cambio total), indicando que la alteración en la forma en que los individuos optan por asistir a un centro público o privado derivó en una distribución menos homogénea. Por último, los resultados indican que el efecto características es el de mayor relevancia, donde los cambios en las características observables e inobservables de los individuos dan cuenta del $66 \%$ del aumento total.

Cuadro 1. Micro descomposiciones. Cambios en el Índice de Disimilitud. Educación primaria y secundaria. Total país

\begin{tabular}{lcccccc}
\hline & \multicolumn{3}{c}{ CAMBIO EN NIVELES } & \multicolumn{3}{c}{ CAMBIO EN \% } \\
\cline { 2 - 7 } & $\mathbf{1 9 9 2 - 2 0 1 7}$ & $\mathbf{1 9 9 2 - 2 0 0 3}$ & $\mathbf{2 0 0 3 - 2 0 1 7}$ & $\mathbf{1 9 9 2 - 2 0 1 7}$ & $\mathbf{1 9 9 2 - 2 0 0 3}$ & $\mathbf{2 0 0 3 - 2 0 1 7}$ \\
\hline Cambio observ. & 0,087 & $-0,042$ & 0,130 & $100 \%$ & $100 \%$ & $100 \%$ \\
\hline Efectos & & & & & & \\
Participación & $-0,004$ & $-0,002$ & $-0,002$ & $-5,0 \%$ & $4,1 \%$ & $-1,5 \%$ \\
Provisión públ. & 0,034 & $-0,002$ & 0,026 & $39,1 \%$ & $4,7 \%$ & $20,3 \%$ \\
Características & 0,058 & $-0,039$ & 0,105 & $65,9 \%$ & $91,2 \%$ & $81,2 \%$ \\
\hline
\end{tabular}

Fuente: Elaboración propia en base a datos de ECH.

Respecto al período 1992-2003, la caída de 0,042 en el índice de Disimilitud se compone de un efecto participación y provisión pública casi despreciables, por lo que el aporte del cambio en las características es el que logra dar cuenta del 90\% del cambio total.

La estimación de la segregación para el período más reciente (2003 - 2017) indica un incremento de 0,13 puntos porcentuales en el índice de Disimilitud. En la columna 3 del cuadro 1 se observa que el $80 \%$ de ese incremento está asociado al efecto características;

\footnotetext{
${ }^{6}$ Los hogares nucleares son aquellos compuestos por hijos viviendo únicamente con ambos padres. Los hogares no nucleares son todos aquellos que no cumplen con la definición anterior.

${ }^{7}$ Debido a que los índices contrafácticos se calculan en base a probabilidades simuladas, los índices observados de esta sección se calcularon utilizando las probabilidades predichas en base a los datos observados de cada año.
} 
ya que el cambio en las características observables e inobservables de los individuos contribuyó a un aumento de 0,105 puntos del índice de segregación. El efecto provisión pública también contribuyó a un incremento de la segregación de 0,026, reflejando que si los parámetros que determinan la elección de educación pública se hubieran mantenido constantes, la distribución hubiera sido más homogénea. Por último, al igual que en los otros dos períodos el efecto participación es prácticamente irrelevante.

Los resultados anteriores sugieren que el efecto participación en la evolución de la segregación fue prácticamente irrelevante. Este resultado es esperable ya que las tasas de asistencia de primaria y secundaria consideradas conjuntamente son similares entre los años. Por su parte, el efecto provisión pública parece haber tenido una relevancia mayor, ya que las diferencias en los parámetros que guían la elección de un centro privado o público dan cuenta de entre un $20 \%$ y un $40 \%$ del aumento de la segregación. El cambio en esos parámetros refleja que, en su decisión de ir a una escuela pública o privada, los individuos están respondiendo en forma distinta frente a los determinantes de dicha elección. Debido a que el signo del efecto provisión pública indica un aumento de la segregación, esas variaciones en los retornos estarían reflejando cambios en las preferencias de los individuos entre el sistema público y privado, en detrimento del primero.

El efecto características parece ser entonces el más relevante para explicar el aumento de la segregación, ya que da cuenta de entre un $65 \%$ y un $80 \%$ del cambio total. La evidencia aportada en la sección anterior sugiere que la segregación aumentó principalmente por una mayor participación de la educación privada en la población no pobre. Varios son los factores que pueden estar detrás de esto. Por ejemplo, si se asume que padres más educados poseen mayor preocupación por la calidad de la educación de sus hijos, los padres más educados migrarían hacia la educación privada si es que perciben mejor calidad en dichas escuelas. Los cambios en la estructura educativa de los padres podrían ser entonces una de las características que inciden en la relevancia del efecto características. También podría suceder que las escuelas privadas sean ahora preferidas en mayor medida debido a que ofrecen servicios más adaptados a las necesidades de las familias. Por ejemplo, una jornada escolar más extensa ofrecida por la educación privada podría ser preferida frente a una mayor participación laboral femenina. En este sentido, cambios en las características laborales de los individuos podrían ser entonces otro de los factores que componen el efecto características.

El aumento del ingreso de los hogares que posibilita el pago de una matrícula en caso de optar por la educación privada o que facilita la migración hacia escuelas privadas aún frente a cambios leves en las preferencias, puede ser otra de las razones detrás del aumento documentado. Si bien pueden nombrarse otros factores que influyen sobre la elección público/privado, un determinado nivel de ingreso es una condición al menos necesaria para optar por la educación privada, en la medida en que ésta implica afrontar el pago de una matrícula. Bajo la hipótesis de que existe una migración de hogares con mayores ingresos hacia la educación privada en busca de un servicio diferenciado, el ingreso se convertiría entonces en un factor relevante en la elección de una escuela pública o privada, afectando la probabilidad de cada una de estas opciones (Gasparini et al., 2011; Jaume, 2013).

Lo anterior se verifica en la figura 6 , donde se muestra la probabilidad de elección de una escuela pública o privada en función del percentil de ingreso per cápita familiar al que 
pertenece el estudiante para ambos niveles educativos considerados en conjunto. El panel izquierdo refiere al nivel primario mientras que el derecho al secundario. La figura refleja dos hechos importantes: i) la probabilidad de optar por la educación pública desciende a medida que crece el ingreso, en particular desde el percentil 10 en adelante; ii) la curva de probabilidad del 2017 se encuentra por debajo de la de 1992, indicando que a igual percentil de ingreso, la probabilidad de elegir la educación pública es menor al final que al comienzo del período.
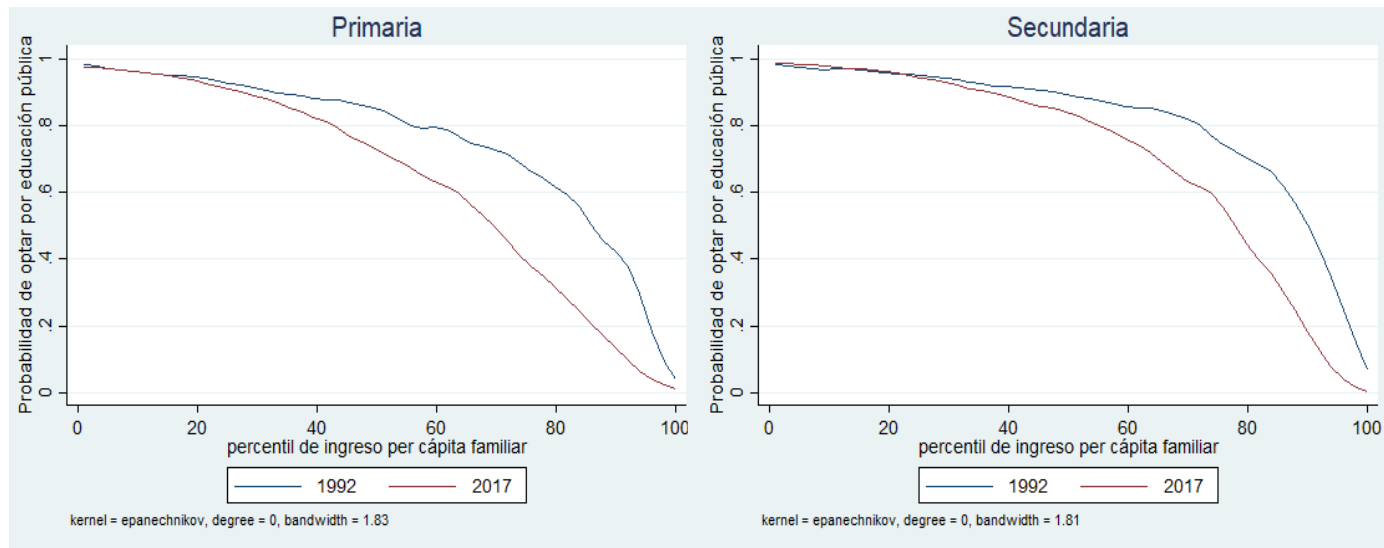

Figura 6. Probabilidad de asistir a la escuela pública en función del percentil de ingreso per cápita familiar. Total país. Años 1992 y 2017

Fuente: Elaboración propia en base a ECH.

Como primer ejercicio a la aproximación de la importancia particular del ingreso, se realiza una simulación de la asistencia y elección de educación pública bajo un escenario donde lo único que cambia entre dos períodos es el ingreso de los hogares, tal como se detalló en la sección metodológica. En base a esto, puede obtenerse una distribución de asistencia público y privada para pobres y no pobres observada $\left(D_{t}\right)$ y una simulada $\left(D_{t x x^{\prime}}\right)$, que surge de cambiar el ingreso de los hogares entre dos momentos del tiempo.

Este ejercicio se realiza considerando los siguientes períodos: 1992-2017; 1992-2003 y 2003-2017. Para cada uno de estos períodos, se calcula cuál hubiese sido el índice de Disimilitud del año de inicio si los ingresos fueran los del año de finalización y se lo compara con el índice de Disimilitud del año de inicio para obtener una primera variación simulada. Luego, se calcula cuál hubiese sido el índice de Disimilitud del año de finalización si los ingresos fueran los del año de inicio y se lo compara con el índice de Disimilitud del año de finalización para obtener una segunda variación simulada. Finalmente, se computa el promedio de las dos variaciones anteriores y se la compara con la variación observada.

El cuadro 2 presenta los resultados que surgen de realizar este ejercicio de simulación de ingresos. Para ello, a cada individuo del momento $t$ se le imputa el ingreso per cápita familiar promedio del año $t^{\prime}$, en función del percentil al que pertenece. En términos generales, se observa que las variaciones en el ingreso de los hogares durante el período analizado son relevantes para explicar las variaciones en la segregación educativa. Previo al análisis de los resultados, es relevante mencionar que este tipo de simulaciones consideran que la estructura condicional de la población se mantiene inalterada, es decir, supone que no se producen efectos de equilibrio general. En este sentido, se aíslan 
comportamientos que son endógenos y se consideran independientes, por lo que las variaciones que se obtienen deben interpretarse como efectos ceteris paribus.

Para el período 1992-2017, los índices de segregación contrafácticos varían en la dirección esperada. Si al año 2017 se le imputan ingresos del año 1992 (menores), la segregación educativa hubiera disminuido 0,056, ceteris paribus, quizás como consecuencia de una mayor participación de la población no pobre en el sistema educativo público; mientras que si a los individuos de 1992 se le imputan los ingresos de 2017 (mayores), la segregación se hubiera incrementado en 0,046 , ceteris paribus; debido posiblemente a que al contar con mayores ingresos los individuos pueden hacer frente al pago de la matrícula que la educación privada requiere, o a que el costo relativo de optar por el sistema privado disminuye, aumentando la participación de la educación privada entre los individuos no pobres.

En los otros dos períodos considerados se encuentran resultados similares. En particular, la simulación de ingresos logra explicar casi un $80 \%$ del aumento de la segregación para el período 2003-2017. Los resultados muestran que si los individuos de este último año hubieran tenido los ingresos del 2003, ceteris paribus, la segregación habría sido 0,118 menor; mientras que si en el 2003 se aplican los ingresos del 2017, la segregación habría sido 0,089 mayor. Lo anterior respalda la idea de que el incremento de la segregación parece estar vinculado en gran medida por el aumento del poder adquisitivo de los hogares, que se traduce en una mayor participación de la enseñanza privada en sectores no pobres.

Cuadro 2. Simulaciones de ingreso. Cambios en el Índice de Disimilitud. Educación primaria y secundaria. Total país

\begin{tabular}{lccc}
\hline & $\mathbf{1 9 9 2 - 2 0 1 7}$ & $\mathbf{1 9 9 2 - 2 0 0 3}$ & $\mathbf{2 0 0 3 - 2 0 1 7}$ \\
\hline Cambio observado & 0,087 & 0,042 & 0,130 \\
\hline Cambio con simulación de ingreso & & & \\
$\quad t$ con ingresos de $t^{\prime}$ & 0,046 & $-0,051$ & 0,089 \\
$\quad t^{\prime}$ con ingresos de $t$ & $-0,056$ & 0,047 & $-0,118$ \\
$\quad$ promedio en niveles & 0,051 & 0,049 & 0,103 \\
$\quad$ promedio como \% del cambio observado & $58,5 \%$ & $115,0 \%$ & $79,6 \%$ \\
\hline
\end{tabular}

Nota: para cada período, $t$ es el año de inicio mientras que $t^{\prime}$ es el año de finalización.

Fuente: Elaboración propia en base ECH.

La microdescomposición realizada sugiere que si bien la evolución de la segregación educativa en Uruguay entre 1992-2017 responde en cierta medida a cambios en los parámetros que gobiernan la elección de la educación pública respecto a la privada, es el efecto características el que mayor importancia ha tenido en la aproximación a los determinantes de la segregación. La simulación que se acaba de realizar, indica que, dentro del conjunto de características de los individuos, el ingreso parecería ser un determinante importante del incremento de la segregación. Para poder concluir sobre este hecho, se combina el ejercicio de simulación con el de micro descomposiciones, esto es, en lugar de realizar la sustitución gradual de parámetros por un lado, y la simulación del ingreso por otro, se realizan ambos cambios conjuntamente. Por tanto, se obtienen indicadores de segregación que surgen de simular cómo hubiera sido la elección de la educación pública de los individuos si los parámetros que gobiernan su decisión de asistencia, los parámetros que determinan su elección público/privado, y su nivel de ingresos, fuesen los de otro momento en el tiempo. 
La fila seis del cuadro 3 confirma la importancia del ingreso per cápita familiar como determinante de los cambios en la segregación, ya que logra explicar un 87\% del total del efecto características y un 57\% del cambio total entre 1992-2017. Cuando la segregación cae (1992-2003), el efecto características parece dar cuenta de casi la totalidad de dicha caída. En este período, los ingresos parecen haber presionado a una caída aún mayor de la segregación, la cual fue contrarrestada por los cambios en otras características. Los cambios en los niveles de ingresos de los individuos parecen haber tenido un rol más determinante aún en los últimos 15 años, ya que dan cuenta de casi la totalidad del efecto características, y por lo tanto de un $79 \%$ del aumento total de la segregación.

Cuadro 3. Micro descomposiciones con simulaciones de ingreso. Cambios en el Índice de Disimilitud. Educación primaria y secundaria. Total país

\begin{tabular}{lcccccc}
\hline & \multicolumn{3}{c}{ CAMBIO EN NIVELES } & \multicolumn{3}{c}{ CAMBIO EN \% } \\
\cline { 2 - 7 } & $\mathbf{1 9 9 2 - 2 0 1 7}$ & $\mathbf{1 9 9 2 - 2 0 0 3}$ & $\mathbf{2 0 0 3 - 2 0 1 7}$ & $\mathbf{1 9 9 2 - 2 0 1 7}$ & $\mathbf{1 9 9 2 - 2 0 0 3}$ & $\mathbf{2 0 0 3 - 2 0 1 7}$ \\
\hline Cambio observ. & 0,087 & $-0,042$ & 0,130 & $100 \%$ & $100 \%$ & $100 \%$ \\
\hline Efectos & & & & & & \\
Participación & $-0,004$ & $-0,002$ & $-0,002$ & $-5,0 \%$ & $4,1 \%$ & $-1,5 \%$ \\
Provisión públ. & 0,034 & $-0,002$ & 0,026 & $39,1 \%$ & $4,7 \%$ & $20,3 \%$ \\
Característ. & 0,058 & $-0,039$ & 0,105 & $65,9 \%$ & $91,2 \%$ & $81,2 \%$ \\
\hline Ingreso & 0,050 & $-0,048$ & 0,103 & $86,7 \%$ & $123,8 \%$ & $97,5 \%$ \\
Otras & 0,008 & 0,009 & 0,003 & $13,3 \%$ & $-23,8 \%$ & $2,5 \%$ \\
\hline Funyyyyy
\end{tabular}

Fuente: Elaboración propia en base a datos de ECH.

\subsection{Educación primaria}

El cuadro 4 muestra los resultados de los ejercicios anteriores para el nivel primario. Se presentan únicamente los resultados del ejercicio combinado de descomposición junto con la simulación de ingreso, ya que este análisis permite evaluar en forma directa qué tan relevantes han sido cada uno de los efectos y la importancia del ingreso dentro del efecto características.

Considerando únicamente al nivel primario, se observa en primer lugar que el efecto participación es más irrelevante aún que cuando se consideraban ambos niveles educativos en conjunto. Esto se explica posiblemente por las tasas de asistencia prácticamente universales que se observan en la educación primaria a lo largo de todo el período de estudio. De este modo, al ser las tasas de asistencia próximas al 100\%, el margen para suponer cambios en el modo en que los individuos deciden asistir es escaso, y por tanto la relevancia de este efecto sobre los cambios en la segregación es prácticamente nula.

El efecto provisión pública no resulta relevante tampoco durante el período donde la segregación disminuye (1992-2003). Sin embargo, adquiere mayor relevancia cuando se evidencia un aumento de la segregación, logrando dar cuenta del $25 \%$ considerando todo el período y un 10\% si se consideran los últimos 15 años.

Del mismo modo que cuando se analizaron ambos niveles educativos conjuntamente, pero con una magnitud aún mayor, el efecto características es el más importante a la hora de descomponer los cambios en la segregación del nivel primario. La diferencia principal que se observa en el análisis de primaria consiste en que si bien el efecto características logra dar cuenta de un mayor porcentaje del cambio, el nivel de ingresos de los individuos es menos relevante dentro de este componente, ya que es un $55 \%$ del efecto características considerando todo el período, y un 75\% de subperíodo 2003-2017. De todos modos, sigue 
siendo un factor determinante: logra dar cuenta del $42 \%$ del aumento total de la segregación en el período 1992-2017 y de un 68\% en los últimos 15 años.

Cuadro 4. Micro descomposiciones con simulaciones de ingreso. Cambios en el Índice de Disimilitud. Educación primaria. Total país

\begin{tabular}{lcccccc}
\hline & \multicolumn{3}{c}{ CAMBIO EN NIVELES } & \multicolumn{3}{c}{ CAMBIO EN \% } \\
\cline { 2 - 7 } & $\mathbf{1 9 9 2 - 2 0 1 7}$ & $\mathbf{1 9 9 2 - 2 0 0 3}$ & $\mathbf{2 0 0 3 - 2 0 1 7}$ & $\mathbf{1 9 9 2 - 2 0 1 7}$ & $\mathbf{1 9 9 2 - 2 0 0 3}$ & $\mathbf{2 0 0 3 - 2 0 1 7}$ \\
\hline Cambio observ. & 0,122 & $-0,036$ & 0,158 & $100 \%$ & $100 \%$ & $100 \%$ \\
\hline Efectos & & & & & & \\
Participación & $-0,001$ & $-0,001$ & $-0,001$ & $-1,0 \%$ & $2,4 \%$ & $-0,6 \%$ \\
Provisión públ. & 0,031 & 0,000 & 0,015 & $25,9 \%$ & $-0,3 \%$ & $9,7 \%$ \\
Características & 0,091 & $-0,036$ & 0,143 & $75,1 \%$ & $97,9 \%$ & $90,9 \%$ \\
\hline Ingreso & 0,051 & $-0,051$ & 0,107 & $55,8 \%$ & $142,2 \%$ & $74,4 \%$ \\
Otras & 0,021 & 0,009 & 0,037 & $44,2 \%$ & $-42,2 \%$ & $25,6 \%$ \\
\hline Funyyyyy
\end{tabular}

Fuente: Elaboración propia en base a datos de ECH.

\subsection{Educación secundaria}

$\mathrm{Al}$ igual que para el nivel primario, el cuadro 5 presenta los resultados que surgen de combinar la micro descomposición con la simulación del ingreso considerando únicamente a los estudiantes de secundaria. Los resultados muestran que existen algunas diferencias respecto al análisis del nivel primario que vale la pena destacar.

En primer lugar, si bien su magnitud sigue siendo escasa, el efecto participación parece tener una relevancia un poco mayor en este nivel educativo, al menos al considerar todo el período en su conjunto. Los resultados sugieren que los cambios en el modo en que los individuos deciden su asistencia compensaron levemente el incremento de la segregación. Esto puede deberse a que en el período considerado, aumentó la preferencia por asistir por parte de los individuos más pobres al sistema educativo, generando por tanto una distribución más homogénea. En segundo lugar, si bien el efecto características sigue siendo el principal, la relevancia del efecto provisión pública es considerablemente mayor que para el nivel primario. Esto sugiere que la segregación en el nivel secundario ha aumentado en el período considerado, entre otras cosas, por cambios en la forma en que los individuos optan entre la educación pública y privada. Esto podría estar reflejando cambios en las preferencias de los individuos, en detrimento de la educación pública, que se producen principalmente en el nivel secundario. ${ }^{8}$ En particular, para el total del período, la relevancia de este efecto da cuenta de la mitad del aumento del índice de Disimilitud. Finalmente, dentro del efecto características (que en comparación con primaria es menor), la relevancia del factor ingreso es mayor. De hecho, en los períodos de aumento, la descomposición indica que el aumento de los ingresos de los individuos hubiera generado un aumento de la segregación aún mayor, lo cual fue contrarrestado por el efecto de otras características.

${ }^{8}$ INEED (2017) indica que en la valoración de la calidad de la educación pública ofrecida en distintos niveles educativos, la educación secundaria pública es la peor evaluada por los encuestados. 
Cuadro 5. Micro descomposiciones con simulaciones de ingreso. Cambios en el Índice de Disimilitud. Educación secundaria. Total país

\begin{tabular}{lcccccc}
\hline & \multicolumn{3}{c}{ CAMBIO EN NIVELES } & \multicolumn{3}{c}{ CAMBIO EN \% } \\
& $\mathbf{1 9 9 2 - 2 0 1 7}$ & $\mathbf{1 9 9 2 - 2 0 0 3}$ & $\mathbf{2 0 0 3 - 2 0 1 7}$ & $\mathbf{1 9 9 2 - 2 0 1 7}$ & $\mathbf{1 9 9 2 - 2 0 0 3}$ & $\mathbf{2 0 0 3 - 2 0 1 7}$ \\
\hline Cambio observ. & 0,072 & $-0,055$ & 0,127 & $100 \%$ & $100 \%$ & $100 \%$ \\
\hline Efectos & & & & & & \\
Participación & $-0,007$ & $-0,004$ & $-0,002$ & $-9,6 \%$ & $6,4 \%$ & $-1,2 \%$ \\
Provisión públ. & 0,037 & $-0,004$ & 0,040 & $51,7 \%$ & $8,1 \%$ & $31,2 \%$ \\
Características & 0,042 & $-0,047$ & 0,089 & $57,8 \%$ & $85,4 \%$ & $70,0 \%$ \\
\hline Ingreso & 0,050 & $-0,046$ & 0,101 & $119,4 \%$ & $98,9 \%$ & $113,4 \%$ \\
Otras & $-0,008$ & $-0,001$ & 0,012 & $-19,4 \%$ & $1,1 \%$ & $13,4 \%$ \\
\hline
\end{tabular}

Fuente: Elaboración propia en base a datos de ECH.

\section{Conclusiones}

Este trabajo propuso una metodología para descomponer los cambios en la segregación escolar en el tiempo y cuantificar la importancia relativa de diferentes factores que dan cuenta de esos cambios. La metodología permite expresar el cambio en la segregación entre dos períodos de tiempo como la suma de un efecto participación, un efecto provisión pública y un efecto características, y descomponer a su vez este último efecto en base a distintos determinantes de la segregación que hayan sido planteados a nivel teórico. Interpretando los dos primeros efectos como resultados de un cambio en las preferencias por la asistencia o por los establecimientos del tipo público, e incorporando al ingreso entre los determinantes de la decisión de asistencia y del tipo de establecimiento elegido, la metodología permite cuantificar de algún modo la importancia relativa de los cambios en la restricción presupuestaria de los individuos en la explicación de los cambios en la segregación.

Para ilustrar la utilidad de la metodología propuesta, se realizó una aplicación empírica al caso de Uruguay, uno de los países de América Latina que mayor incremento de la segregación escolar entre escuelas públicas y privadas ha experimentado en los últimos años. Los resultados hallados muestran que los cambios en las preferencias por asistir a la escuela dan cuenta de una fracción muy pequeña del aumento de la segregación ocurrido en este país entre 1992 y 2017 . En cambio, las preferencias por asistir a un establecimiento del tipo privado tuvieron un rol importante en el cambio observado en la segregación, particularmente en secundaria, mientras que los cambios en la restricción presupuestaria, captados vía modificaciones en los ingresos, explican la mayor parte del cambio observado en la segregación durante el período.

Por limitaciones en los datos disponibles, los cambios en los precios relativos de los establecimientos públicos y privados no pudieron incorporarse en la aplicación empírica, pero la metodología es lo suficientemente flexible para incorporar esta parte importante de la restricción presupuestaria al modelo, así como también el efecto de los cambios en la segregación residencial si se incorpora información sobre la localización geográfica de los individuos. Finalmente, si se asignaran características de las escuelas a los individuos de algún modo, podría cuantificarse cuánto de lo que aquí se interpreta como cambio en las preferencias en un sentido amplio se refiere a cambios en las características de las escuelas que los individuos valoran y tienen en cuenta al tomar su decisión de asistir a un establecimiento escolar. 
Aunque las limitaciones de la metodología impiden hablar de causalidad y se trata de un ejercicio de equilibrio parcial, los resultados encontrados señalan que al menos en el caso uruguayo, cualquier explicación teórica de los cambios en la segregación acontecidos durante el período debería otorgarle importancia a los cambios ocurridos en los ingresos de los individuos. Al ayudar a desentrañar el aporte relativo de diferentes teorías que pueden dar cuenta de los cambios observados en la segregación, la metodología propuesta constituye una herramienta útil para enriquecer el análisis de sus determinantes, complementando otros enfoques que puedan realizarse y contribuyendo a una mejor comprensión de los cambios experimentados en la segregación escolar.

\section{Referencias}

Arcidiácono, M., Cruces, G., Gasparini, L., Jaume, D., Serio, M. y Vazquez, E. (2014). La segregación escolar público-privado en América Latina. Serie Políticas Sociales de CEPAL, $195(1), 1-35$.

Becker, G. S. y Murphy, K. M. (2003). Social economics: Market behavior in a social environment. Belknap Press.

Bourguignon, F., Ferreira, F. y Lustig, N. (2005). The microeconomics of income distribution dynamics in East Asia and Latin America. World Bank.

Card, D., Mas, A. y Rothstein, J. (2008). Tipping and the dynamics of segregation. The Quarterly Journal of Economics, 123(1), 177-218. https://doi.org/10.1162/qjec.2008.123.1.177

Duncan, O. y Duncan, B. (1955). A methodological analysis of segregation indexes. American Sociological Review, 20(2), 210-217.

Durlauf, S. (2006). Groups, social influences, and inequality: A memberships theory perspective on poverty traps. En S. Bowles, S. Durlauf y K. Hoff (Eds.), Poverty traps (pp. 141-175). Princeton University Press.

Epple, D. y Romano, R. E. (1998). Competition between private and public schools, vouchers, and peer-group effects. American Economic Review, 88(1), 33-62.

Fortin, N., Lemieux, T. y Firpo, S. (2011). Decomposition Method in Economics. En O. Ashenfelter y D. Card (Eds.), Handbook of labor economics (pp. 11-102). Elsevier.

Gasparini, L. (2006). Assessing benefit-incidence results using decompositions. The case of health policy in Argentina. Economics Bulletin, 4(40), 1-10.

Gasparini, L., Jaume, D., Serio, M. y Vazquez, E. (2011). La segregación escolar entre escuelas públicas y privadas en Argentina. Reconstruyendo la evidencia. Desarrollo Económico-Revista de Ciencias Sociales, 51(202-203), 189-219.

Jaume, D. (2013). Un estudio sobre el incremento de la segregación escolar en Argentina. Serie Documentos de trabajo del CEDLAS, 143, 1-43.

Jenkins, S., Micklewright, J. y Schnepf, S. (2008). Social segregation in secondary schools: How does England compare with other countries? Oxford Review of Education, 34(1), 21-38. https://doi.org/10.1080/03054980701542039

Murillo, F. J. (2016). Midiendo la segregación escolar en América Latina. Un análisis metodológico utilizando el TERCE. REICE. Revista Iberoamericana sobre Calidad, Eficacia y Cambio en Educación, 14(4), 33-60. https://doi.org/10.15366/reice2016.14.4.002 
Murillo, F. J. y Martínez-Garrido, C. (2017). Estimación de la magnitud de la segregación escolar en América Latina. Magis. Revista Internacional de Investigación en Educación, 9(19), 11-30. https://doi.org/10.11144/javeriana.m9-19.emse

Murillo, F. J., Belavi, G. y Pinilla, L. (2018). Segregación escolar público-privada en España. Papers, 103(3), 307-337. https://doi.org/10.5565/rev/papers.2392

Nechyba, T. J. (2006). Income and peer quality sorting in public and private schools. En E. Hanushek y F. Welch (Eds.), Handbook of the economics of education (pp. 1327-1368). North Holland.

Schelling, T. C. (1971). Dynamic models of segregation. Journal of Mathematical Sociology, 1(2), 143-186. https://doi.org/10.1080/0022250X.1971.9989794

Valenzuela, J. P. (2008). Evolución de la segregación socioeconómica de los estudiantes chilenos y su relación con el financiamiento compartido. Ministerio de Educación de Chile.

Vazquez, E. (2012). Segregación escolar por nivel socioeconómico. Midiendo el fenómeno y explorando sus determinantes. Serie Documentos de trabajo del CEDLAS, 128, 1-35.

Vazquez, E. (2016). Segregación escolar por nivel socioeconómico. Midiendo el fenómeno y explorando sus determinantes. Económica, 42, 121-184.

Wilson, W. (1987). The truly disadvantaged: The inner city, the underclass, and public policy. University of Chicago Press. 


\section{Anexo}

Cuadro A1. Evolución del ingreso per cápita familiar por quintiles de ingreso. Total nacional 1992, 2003, 2017

\begin{tabular}{lcccccc}
\hline & \multicolumn{3}{c}{ EN NIVELES } & \multicolumn{3}{c}{ EN \% } \\
\cline { 2 - 7 } & $\mathbf{1 9 9 2}$ & $\mathbf{2 0 0 3}$ & $\mathbf{2 0 1 7}$ & $\mathbf{1 9 9 2 - 2 0 1 7}$ & $\mathbf{1 9 9 2 - 2 0 0 3}$ & $\mathbf{2 0 0 3 - 2 0 1 7}$ \\
\hline Quintil 1 & 1939 & 1333 & 2654 & $36,9 \%$ & $-31,2 \%$ & $99,0 \%$ \\
Quintil 2 & 3667 & 2343 & 4856 & $32,4 \%$ & $-36,1 \%$ & $107,3 \%$ \\
Quintil 3 & 5493 & 3603 & 7110 & $29,5 \%$ & $-34,4 \%$ & $97,4 \%$ \\
Quintil 4 & 8090 & 5569 & 10334 & $27,7 \%$ & $-31,2 \%$ & $85,6 \%$ \\
Quintil 5 & 17392 & 13253 & 21118 & $21,4 \%$ & $\mathbf{- 2 3 , 8} \%$ & $59,3 \%$ \\
Promedio & 7316 & 5220 & 9215 & $26,0 \%$ & $\mathbf{- 2 8 , 6} \%$ & $76,5 \%$ \\
Mediana & 5471 & 3576 & 7069 & $29,2 \%$ & $\mathbf{- 3 4 , 6} \%$ & $97,7 \%$ \\
\hline
\end{tabular}

Nota: Ingreso per cápita familiar a pesos constantes de 2005. Metodología CEDLAS.

Fuente: Elaboración propia en base a ECH.

Cuadro A2. Descripción de las variables empleadas en los modelos

\begin{tabular}{ll}
\hline NombRE & \\
\hline hombre & $1=$ Hombre \\
edad & Años de edad cumplidos \\
nuclear & $1=$ Hogar nuclear (hijos viviendo únicamente con ambos padres) \\
hnos1 & $1=$ El individuo tiene un hermano \\
hnos2 & $1=$ El individuo tiene dos hermanos \\
hnos3 & $1=$ El individuo tiene tres hermanos \\
hnos4 & $1=$ El individuo tiene cuatro o más hermanos \\
hijo2 & $1=$ El individuo es el segundo hijo en orden de nacimiento \\
hijo3 & $1=$ El individuo es el tercer hijo en orden de nacimiento \\
hijo4 & $1=$ El individuo es el cuarto hijo en orden de nacimiento (o más) \\
pric_padres & $1=$ El máximo nivel educativo de los padres es primaria completa \\
seci_padres & $1=$ El máximo nivel educativo de los padres es secundaria incompleta \\
secc_padres & $1=$ El máximo nivel educativo de los padres es secundaria completa \\
supi_padres & $1=$ El máximo nivel educativo de los padres es superior incompleto \\
supc_padres & $1=$ El máximo nivel educativo de los padres es superior completo \\
ocupado_jefe & $1=$ El jefe del hogar está ocupado (tiene empleo) \\
dos_pea & $1=$ El jefe del hogar y su cónyuge pertenecen ambos a la población \\
metrop & $\begin{aligned} 1 \\
\text { económicamente activa }\end{aligned}$ \\
ipcf & Ingrea metropolitana per cápita del hogar \\
\hline
\end{tabular}

Fuente: Elaboración propia en base a ECH. 
Cuadro A3. Probabilidad de asistencia y elección de educación pública. Total educación básica. 1992, 2003 y 2017

\section{$199202003 \quad 2017$}

Asistencia Ed. Pública Asistencia Ed. Pública Asistencia Ed. Pública

\begin{tabular}{|c|c|c|c|c|c|c|}
\hline hombre & $\begin{array}{c}-0,277^{* * *} \\
(0,0417)\end{array}$ & $\begin{array}{c}0,107 * * * \\
(0,0393)\end{array}$ & $\begin{array}{r}-0,172^{* * * *} \\
(0,00701) \\
\end{array}$ & $\begin{array}{c}-0,0204^{* * * *} \\
(0,00681)\end{array}$ & $\begin{array}{l}-0,133 * * * \\
(0,00796)\end{array}$ & $\begin{array}{c}0,0566^{* * * *} \\
(0,00624)\end{array}$ \\
\hline edad & $\begin{array}{l}-0,228^{* * *} \\
(0,00812) \\
\end{array}$ & $\begin{array}{c}0,0524 * * * \\
(0,00613) \\
\end{array}$ & $\begin{array}{l}-0,179^{* * *} \\
(0,00125) \\
\end{array}$ & $\begin{array}{c}0,0440^{* * *} * \\
(0,00103)\end{array}$ & $\begin{array}{r}-0,226^{* * *} * \\
(0,00185) \\
\end{array}$ & $\begin{array}{l}0,0506^{* * * *} \\
(0,000900)\end{array}$ \\
\hline nuclear & $\begin{array}{r}-0,00585 \\
(0,0616) \\
\end{array}$ & $\begin{array}{l}-0,0593 \\
(0,0716)\end{array}$ & $\begin{array}{c}-0,130 \text { *** } \\
(0,0102) \\
\end{array}$ & $\begin{array}{c}-0,115^{* * * *} \\
(0,0121) \\
\end{array}$ & $\begin{array}{c}0,149^{* * *} * \\
(0,0122)\end{array}$ & $\begin{array}{c}-0,236^{* * * *} \\
(0,0116) \\
\end{array}$ \\
\hline hnos 1 & $\begin{array}{c}0,358 * * * \\
(0,0643)\end{array}$ & $\begin{array}{c}-0,155^{* *} \\
(0,0634)\end{array}$ & $\begin{array}{c}0,177^{* * * *} \\
(0,0103)\end{array}$ & $\begin{array}{c}0,0101 \\
(0,0103)\end{array}$ & $\begin{array}{c}0,223^{* * * *} \\
(0,0115)\end{array}$ & $\begin{array}{c}-0,117^{* * * *} \\
(0,00883) \\
\end{array}$ \\
\hline hnos2 & $\begin{array}{c}0,234 * * * \\
(0,0628)\end{array}$ & $\begin{array}{c}-0,215^{* * *} \\
(0,0682)\end{array}$ & $\begin{array}{c}0,188^{* * * *} \\
(0,0111)\end{array}$ & $\begin{array}{c}-0,00766 \\
(0,0119)\end{array}$ & $\begin{array}{c}0,163^{* * *} \\
(0,0127)\end{array}$ & $\begin{array}{c}-0,0821 * * * \\
(0,0115)\end{array}$ \\
\hline hnos3 & $\begin{array}{c}0,119^{*} \\
(0,0673)\end{array}$ & $\begin{array}{l}-0,0864 \\
(0,0811)\end{array}$ & $\begin{array}{c}0,123^{* * * *} \\
(0,0121)\end{array}$ & $\begin{array}{c}0,0527 * * * \\
(0,0149)\end{array}$ & $\begin{array}{c}0,135^{* * * *} \\
(0,0152)\end{array}$ & $\begin{array}{c}-0,0596^{* * *} \\
(0,0165)\end{array}$ \\
\hline hnos4 & $\begin{array}{l}-0,0982 \\
(0,0796)\end{array}$ & $\begin{array}{c}0,120 \\
(0,131)\end{array}$ & $\begin{array}{c}-0,167 * * * \\
(0,0145)\end{array}$ & $\begin{array}{c}0,214 * * * \\
(0,0251)\end{array}$ & $\begin{array}{c}0,124^{* * *} \\
(0,0187)\end{array}$ & $\begin{array}{c}0,429 * * * \\
(0,0343)\end{array}$ \\
\hline hijo2 & $\begin{array}{c}-0,158^{* * *} \\
(0,0509)\end{array}$ & $\begin{array}{c}0,182^{* * * *} \\
(0,0470)\end{array}$ & $\begin{array}{l}-0,123 * * * \\
(0,00834)\end{array}$ & $\begin{array}{c}0,0856 * * * \\
(0,00826)\end{array}$ & $\begin{array}{r}-0,152^{* * * *} \\
(0,00958)\end{array}$ & $\begin{array}{l}0,117 * * * \\
(0,00766)\end{array}$ \\
\hline hijo3 & $\begin{array}{c}-0,107 \\
(0,0653)\end{array}$ & $\begin{array}{c}0,337 * * * \\
(0,0687)\end{array}$ & $\begin{array}{c}-0,106^{* * *} \\
(0,0114) \\
\end{array}$ & $\begin{array}{c}0,197 * * * \\
(0,0127)\end{array}$ & $\begin{array}{c}-0,170^{* * *} \\
(0,0137) \\
\end{array}$ & $\begin{array}{c}0,146^{* * * *} \\
(0,0138)\end{array}$ \\
\hline hijo4 & $\begin{array}{l}-0,134^{*} \\
(0,0747)\end{array}$ & $\begin{array}{c}0,378^{* * * *} \\
(0,0942) \\
\end{array}$ & $\begin{array}{l}-0,0206 \\
(0,0142)\end{array}$ & $\begin{array}{c}0,282^{* * * *} \\
(0,0185) \\
\end{array}$ & $\begin{array}{c}-0,0486^{* *} \\
(0,0208) \\
\end{array}$ & $\begin{array}{c}0,173^{* * *} * \\
(0,0229)\end{array}$ \\
\hline pric_padres & $\begin{array}{r}-0,00219 \\
(0,0452)\end{array}$ & $\begin{array}{c}0,279^{* * * *} \\
(0,0507)\end{array}$ & $\begin{array}{c}0,00855 \\
(0,00857) \\
\end{array}$ & $\begin{array}{c}0,461^{* * * *} \\
(0,0111)\end{array}$ & $\begin{array}{c}-0,0436^{* * * *} \\
(0,0108)\end{array}$ & $\begin{array}{c}0,566^{* * * *} \\
(0,0111) \\
\end{array}$ \\
\hline seci_padres & $\begin{array}{c}0,375^{* * * *} \\
(0,0464)\end{array}$ & $\begin{array}{c}-0,138^{* * * *} \\
(0,0481)\end{array}$ & $\begin{array}{l}0,262 * * * \\
(0,00834)\end{array}$ & $\begin{array}{l}0,247 * * * \\
(0,00932)\end{array}$ & $\begin{array}{c}0,274^{* * * *} \\
(0,0107)\end{array}$ & $\begin{array}{l}0,172 * * * \\
(0,00921)\end{array}$ \\
\hline secc_padres & $\begin{array}{c}0,519^{* *} \\
(0,209)\end{array}$ & $\begin{array}{r}-0,0989 \\
(0,126) \\
\end{array}$ & $\begin{array}{c}0,466^{* * * *} \\
(0,0129) \\
\end{array}$ & $\begin{array}{r}-0,167 * * * \\
(0,00903) \\
\end{array}$ & $\begin{array}{c}0,401 * * * \\
(0,0148)\end{array}$ & $\begin{array}{c}-0,240^{* * * *} \\
(0,00851) \\
\end{array}$ \\
\hline supi_padres & $\begin{array}{c}0,775^{* * * *} \\
(0,164)\end{array}$ & $\begin{array}{c}-0,345^{* * *} * \\
(0,0628) \\
\end{array}$ & $\begin{array}{c}0,235 * * * \\
(0,0135) \\
\end{array}$ & $\begin{array}{r}-0,314^{* * * *} \\
(0,00929) \\
\end{array}$ & $\begin{array}{c}0,332 * * * \\
(0,0200)\end{array}$ & $\begin{array}{c}-0,392^{* * * *} \\
(0,00970) \\
\end{array}$ \\
\hline supc_padres & $\begin{array}{c}0,876^{* * * *} \\
(0,144)\end{array}$ & $\begin{array}{c}-0,401 * * * \\
(0,0602)\end{array}$ & $\begin{array}{c}0,505^{* * * *} \\
(0,0190)\end{array}$ & $\begin{array}{c}-0,473^{* * * *} \\
(0,0103)\end{array}$ & $\begin{array}{c}0,265^{* * *} \\
(0,0185)\end{array}$ & $\begin{array}{l}-0,397 * * * \\
(0,00984)\end{array}$ \\
\hline ocupado_jefe & $\begin{array}{l}-0,0596 \\
(0,0682)\end{array}$ & $\begin{array}{r}-0,00565 \\
(0,0928) \\
\end{array}$ & $\begin{array}{l}0,0194 * * \\
(0,00984) \\
\end{array}$ & $\begin{array}{c}-0,227 * * * \\
(0,0129) \\
\end{array}$ & $\begin{array}{c}0,160 * * * * \\
(0,0111) \\
\end{array}$ & $\begin{array}{l}0,00782 \\
(0,0119)\end{array}$ \\
\hline dos_pea & $\begin{array}{c}0,266 * * * \\
(0,0464) \\
\end{array}$ & $\begin{array}{c}0,142^{* * * *} \\
(0,0441) \\
\end{array}$ & $\begin{array}{l}0,101 * * * \\
(0,00820) \\
\end{array}$ & $\begin{array}{c}-0,0589 * * * \\
(0,00892)\end{array}$ & $\begin{array}{l}-0,0102 \\
(0,0116)\end{array}$ & $\begin{array}{c}0,0410^{* * * *} \\
(0,00967) \\
\end{array}$ \\
\hline metrop & $\begin{array}{c}0,0179 \\
(0,0431)\end{array}$ & $\begin{array}{c}-0,672^{* * * *} \\
(0,0455)\end{array}$ & $\begin{array}{c}-0,105 * * * \\
(0,00712) \\
\end{array}$ & $\begin{array}{c}-0,690^{* * *} \\
(0,00821)\end{array}$ & $\begin{array}{l}-0,00173 \\
(0,00810)\end{array}$ & $\begin{array}{c}-0,562 * * * \\
(0,00681) \\
\end{array}$ \\
\hline ipcf & $\begin{array}{c}6,94 \mathrm{e}-05^{* * * *} \\
(8,22 \mathrm{e}-06)\end{array}$ & $\begin{array}{c}-0,000114^{* * * *} \\
(4,34 \mathrm{e}-06)\end{array}$ & $\begin{array}{c}7,03 \mathrm{e}-05^{* * * *} \\
(1,96 \mathrm{e}-06)\end{array}$ & $\begin{array}{c}-0,000115^{* * * *} \\
(8,54 \mathrm{e}-07)\end{array}$ & $\begin{array}{c}3,76 \mathrm{e}-05^{* * * *} \\
(1,35 \mathrm{e}-06)\end{array}$ & $\begin{array}{c}-0,000146^{* * * *} \\
(8,80 \mathrm{e}-07)\end{array}$ \\
\hline Constante & $\begin{array}{c}3,741 * * * \\
(0,151)\end{array}$ & $\begin{array}{c}1,643^{* * * *} \\
(0,141)\end{array}$ & $\begin{array}{c}3,478 * * * \\
(0,0239)\end{array}$ & $\begin{array}{l}1,997 * * * \\
(0,0218)\end{array}$ & $\begin{array}{l}4,179^{* * * *} \\
(0,0328)\end{array}$ & $\begin{array}{l}1,824 * * * \\
(0,0203)\end{array}$ \\
\hline Obs & 9,799 & 8,599 & 386,378 & 358,843 & 451,601 & 341,288 \\
\hline
\end{tabular}

Nota: Estimaciones Probit. Individuos entre 6 a 17 años que asisten a educación primaria o secundaria para el total del país.

Fuente: Elaboración propia en base a ECH. 
Cuadro A4. Probabilidad de asistencia y elección de educación pública. Educación primaria. Total país. 1992, 2003 y 2017

\begin{tabular}{|c|c|c|c|c|c|c|}
\hline & \multicolumn{2}{|c|}{1992} & \multicolumn{2}{|c|}{2003} & \multicolumn{2}{|c|}{2017} \\
\hline & Asistencia & Ed. Pública & Asistencia & Ed. Pública & Asistencia & Ed. Pública \\
\hline hombre & $\begin{array}{l}-0,0806 \\
(0,0917)\end{array}$ & $\begin{array}{c}0,0716 \\
(0,0537) \\
\end{array}$ & $\begin{array}{c}0,0914 * * * \\
(0,0131) \\
\end{array}$ & $\begin{array}{c}0,00956 \\
(0,00942) \\
\end{array}$ & $\begin{array}{c}0,0201 \\
(0,0198)\end{array}$ & $\begin{array}{c}0,117 * * * \\
(0,0100)\end{array}$ \\
\hline edad & $\begin{array}{c}0,0882^{* * * *} \\
(0,0230)\end{array}$ & $\begin{array}{c}0,0454^{* * * *} \\
(0,0148) \\
\end{array}$ & $\begin{array}{c}-0,0348^{* * *} * \\
(0,00331) \\
\end{array}$ & $\begin{array}{c}0,0294 * * * \\
(0,00256) \\
\end{array}$ & $\begin{array}{c}-0,105 * * * \\
(0,00536) \\
\end{array}$ & $\begin{array}{l}0,00621 * * \\
(0,00264) \\
\end{array}$ \\
\hline nuclear & $\begin{array}{l}0,0825 \\
(0,139) \\
\end{array}$ & $\begin{array}{l}0,0179 \\
(0,106) \\
\end{array}$ & $\begin{array}{c}-0,260 * * * \\
(0,0224)\end{array}$ & $\begin{array}{c}-0,108^{* * *} \\
(0,0175) \\
\end{array}$ & $\begin{array}{l}-0,0284 \\
(0,0316)\end{array}$ & $\begin{array}{c}-0,292 * * * \\
(0,0189)\end{array}$ \\
\hline hnos 1 & $\begin{array}{c}0,264 \\
(0,165)\end{array}$ & $\begin{array}{c}-0,249^{* * * *} \\
(0,0910)\end{array}$ & $\begin{array}{c}0,0317 \\
(0,0197)\end{array}$ & $\begin{array}{c}0,0895 * * * \\
(0,0139) \\
\end{array}$ & $\begin{array}{c}0,176^{* * * *} \\
(0,0323)\end{array}$ & $\begin{array}{l}0,00494 \\
(0,0145)\end{array}$ \\
\hline hnos2 & $\begin{array}{c}0,198 \\
(0,153)\end{array}$ & $\begin{array}{c}-0,230^{* *} \\
(0,0987)\end{array}$ & $\begin{array}{c}0,201 * * * \\
(0,0226)\end{array}$ & $\begin{array}{c}0,0949^{* * * *} \\
(0,0166)\end{array}$ & $\begin{array}{c}0,0193 \\
(0,0359)\end{array}$ & $\begin{array}{c}0,0775^{* * *} \\
(0,0194) \\
\end{array}$ \\
\hline hnos3 & $\begin{array}{c}-0,0586 \\
(0,139)\end{array}$ & $\begin{array}{l}-0,155 \\
(0,115)\end{array}$ & $\begin{array}{c}0,134 * * * \\
(0,0252)\end{array}$ & $\begin{array}{c}0,203 * * * \\
(0,0207)\end{array}$ & $\begin{array}{l}0,0731^{*} \\
(0,0442)\end{array}$ & $\begin{array}{c}-0,0633^{*} * \\
(0,0257) \\
\end{array}$ \\
\hline hnos4 & $\begin{array}{c}-0,0230 \\
(0,167)\end{array}$ & $\begin{array}{c}0,135 \\
(0,186)\end{array}$ & $\begin{array}{c}0,0542 \\
(0,0344)\end{array}$ & $\begin{array}{c}0,488 * * * \\
(0,0377)\end{array}$ & $\begin{array}{c}0,217 * * * \\
(0,0676)\end{array}$ & $\begin{array}{c}0,502 * * * \\
(0,0525)\end{array}$ \\
\hline hijo2 & $\begin{array}{c}0,104 \\
(0,133) \\
\end{array}$ & $\begin{array}{l}0,138 * * \\
(0,0637)\end{array}$ & $\begin{array}{c}-0,100 * * * \\
(0,0157) \\
\end{array}$ & $\begin{array}{c}0,102 * * * \\
(0,0118)\end{array}$ & $\begin{array}{c}-0,352^{* * * *} \\
(0,0257)\end{array}$ & $\begin{array}{c}0,0667^{* * *} * \\
(0,0125) \\
\end{array}$ \\
\hline hijo3 & $\begin{array}{l}-0,113 \\
(0,141)\end{array}$ & $\begin{array}{c}0,364 * * * \\
(0,0949)\end{array}$ & $\begin{array}{c}0,107 * * * \\
(0,0237)\end{array}$ & $\begin{array}{c}0,148^{* * *} \\
(0,0176)\end{array}$ & $\begin{array}{r}-0,00523 \\
(0,0402)\end{array}$ & $\begin{array}{c}0,196^{* * * *} \\
(0,0218)\end{array}$ \\
\hline hijo4 & $\begin{array}{r}-0,0256 \\
(0,144) \\
\end{array}$ & $\begin{array}{c}0,428 * * * \\
(0,122) \\
\end{array}$ & $\begin{array}{c}0,256^{* * * *} \\
(0,0280)\end{array}$ & $\begin{array}{c}0,206^{* * *} \\
(0,0225) \\
\end{array}$ & $\begin{array}{l}-0,101^{* *} \\
(0,0511)\end{array}$ & $\begin{array}{c}0,395^{* * * *} \\
(0,0322)\end{array}$ \\
\hline pric_padres & $\begin{array}{l}-0,107 \\
(0,103)\end{array}$ & $\begin{array}{c}0,196 * * * \\
(0,0687)\end{array}$ & $\begin{array}{c}0,220 * * * \\
(0,0174)\end{array}$ & $\begin{array}{c}0,439 * * * \\
(0,0150)\end{array}$ & $\begin{array}{l}-0,0277 \\
(0,0302)\end{array}$ & $\begin{array}{c}0,575^{* * * *} \\
(0,0174)\end{array}$ \\
\hline seci_padres & $\begin{array}{c}0,124 \\
(0,103) \\
\end{array}$ & $\begin{array}{l}-0,127^{*} \\
(0,0659)\end{array}$ & $\begin{array}{r}-0,00409 \\
(0,0167) \\
\end{array}$ & $\begin{array}{c}0,195 * * * \\
(0,0127)\end{array}$ & $\begin{array}{c}0,0714^{* *} \\
(0,0282)\end{array}$ & $\begin{array}{c}0,148^{* * *} \\
(0,0144) \\
\end{array}$ \\
\hline secc_padres & $\begin{array}{c}0,243 \\
(0,370)\end{array}$ & $\begin{array}{l}-0,122 \\
(0,152) \\
\end{array}$ & $\begin{array}{c}0,172 * * * \\
(0,0205)\end{array}$ & $\begin{array}{c}-0,257^{* * *} \\
(0,0124) \\
\end{array}$ & $\begin{array}{c}-0,139 * * * \\
(0,0288)\end{array}$ & $\begin{array}{c}-0,236 * * * \\
(0,0136) \\
\end{array}$ \\
\hline supi_padres & $\begin{array}{l}0,0724 \\
(0,273) \\
\end{array}$ & $\begin{array}{c}-0,434^{* * * *} \\
(0,0870) \\
\end{array}$ & $\begin{array}{c}-0,175^{* * *} \\
(0,0196) \\
\end{array}$ & $\begin{array}{c}-0,329^{* * *} \\
(0,0128) \\
\end{array}$ & $\begin{array}{c}-0,128^{* * * *} \\
(0,0326)\end{array}$ & $\begin{array}{c}-0,330 * * * \\
(0,0152) \\
\end{array}$ \\
\hline supc_padres & $\begin{array}{c}0,281 \\
(0,336) \\
\end{array}$ & $\begin{array}{c}-0,321^{* * * *} \\
(0,0855) \\
\end{array}$ & $\begin{array}{c}0,269 * * * \\
(0,0279) \\
\end{array}$ & $\begin{array}{c}-0,624^{* * * *} \\
(0,0143) \\
\end{array}$ & $\begin{array}{c}-0,225^{* * * *} \\
(0,0334)\end{array}$ & $\begin{array}{c}-0,480^{* * * *} \\
(0,0158) \\
\end{array}$ \\
\hline ocupado_jefe & $\begin{array}{l}0,0525 \\
(0,156)\end{array}$ & $\begin{array}{c}0,00884 \\
(0,138)\end{array}$ & $\begin{array}{c}-0,125^{* * *} * \\
(0,0221)\end{array}$ & $\begin{array}{c}-0,471^{* * * *} \\
(0,0193)\end{array}$ & $\begin{array}{c}-0,426^{* * * *} \\
(0,0445)\end{array}$ & $\begin{array}{l}-0,0220 \\
(0,0185)\end{array}$ \\
\hline dos_pea & $\begin{array}{c}0,371^{* * * *} \\
(0,107)\end{array}$ & $\begin{array}{c}0,0676 \\
(0,0596)\end{array}$ & $\begin{array}{c}0,162 * * * \\
(0,0148)\end{array}$ & $\begin{array}{c}-0,0370^{* * *} \\
(0,0120)\end{array}$ & $\begin{array}{c}0,196 * * * \\
(0,0274)\end{array}$ & $\begin{array}{l}-0,0228 \\
(0,0149)\end{array}$ \\
\hline metrop & $\begin{array}{c}0,0526 \\
(0,0953) \\
\end{array}$ & $\begin{array}{c}-0,617^{* * * *} \\
(0,0604) \\
\end{array}$ & $\begin{array}{c}-0,0310^{* *} \\
(0,0136) \\
\end{array}$ & $\begin{array}{c}-0,586^{* * * *} \\
(0,0111) \\
\end{array}$ & $\begin{array}{c}-0,0611^{* * *} * \\
(0,0209)\end{array}$ & $\begin{array}{c}-0,581^{* * * *} \\
(0,0108) \\
\end{array}$ \\
\hline ipcf & $\begin{array}{l}6,38 \mathrm{e}-05^{* *} \\
(2,48 \mathrm{e}-05) \\
\end{array}$ & $\begin{array}{c}-0,000117^{*} * * \\
(6,56 \mathrm{e}-06)\end{array}$ & $\begin{array}{c}9,22 \mathrm{e}-06 \text { **** } \\
(2,39 \mathrm{e}-06) \\
\end{array}$ & $\begin{array}{c}-0,000124^{* * * *} \\
(1,30 \mathrm{e}-06)\end{array}$ & $\begin{array}{c}-1,78 \mathrm{e}-06 \\
(2,04 \mathrm{e}-06)\end{array}$ & $\begin{array}{c}-0,000139^{* * * *} * \\
(1,42 \mathrm{e}-06)\end{array}$ \\
\hline Constante & $\begin{array}{c}0,751^{* *} \\
(0,322)\end{array}$ & $\begin{array}{c}1,765^{* * * *} \\
(0,225)\end{array}$ & $\begin{array}{c}2,422^{* * * *} \\
(0,0454)\end{array}$ & $\begin{array}{c}2,308^{* * *} \\
(0,0346)\end{array}$ & $\begin{array}{l}4,011^{* * * *} \\
(0,0807)\end{array}$ & $\begin{array}{c}2,198^{* * *} \\
(0,0355)\end{array}$ \\
\hline Obs & 5,356 & 4,587 & 216,762 & 193,325 & 243,31 & 125,81 \\
\hline
\end{tabular}

Nota: Estimaciones Probit. Individuos entre 6 a 11 años que asisten a educación primaria para el total del país.

Fuente: Elaboración propia en base a datos de ECH. 
Cuadro A5. Probabilidad de asistencia y elección de educación pública. Educación secundaria. Total país. 1992, 2003 y 2017

\begin{tabular}{|c|c|c|c|c|c|c|}
\hline & \multicolumn{2}{|c|}{1992} & \multicolumn{2}{|c|}{2003} & \multicolumn{2}{|c|}{2017} \\
\hline & Asistencia & Ed. Pública & Asistencia & Ed. Pública & Asistencia & Ed. Pública \\
\hline hombre & $\begin{array}{c}-0,330^{* * * *} \\
(0,0469) \\
\end{array}$ & $\begin{array}{l}0,134 * * \\
(0,0597)\end{array}$ & $\begin{array}{c}-0,258^{* * * *} \\
(0,00812) \\
\end{array}$ & $\begin{array}{c}-0,0474^{* * * *} \\
(0,0102)\end{array}$ & $\begin{array}{r}-0,166^{* * *} \\
(0,00842) \\
\end{array}$ & $\begin{array}{l}0,0184 * * \\
(0,00812) \\
\end{array}$ \\
\hline edad & $\begin{array}{c}-0,362 * * * \\
(0,0144) \\
\end{array}$ & $\begin{array}{c}0,131 * * * \\
(0,0182) \\
\end{array}$ & $\begin{array}{c}-0,288^{* * * *} \\
(0,00233) \\
\end{array}$ & $\begin{array}{c}0,0958 * * * \\
(0,00307) \\
\end{array}$ & $\begin{array}{r}-0,279^{* * *} \\
(0,00264) \\
\end{array}$ & $\begin{array}{l}0,130 * * * \\
(0,00248) \\
\end{array}$ \\
\hline nuclear & $\begin{array}{c}0,0230 \\
(0,0682) \\
\end{array}$ & $\begin{array}{l}-0,147 \\
(0,101) \\
\end{array}$ & $\begin{array}{c}-0,116^{* * * *} \\
(0,0115) \\
\end{array}$ & $\begin{array}{c}-0,158^{* * * *} \\
(0,0175) \\
\end{array}$ & $\begin{array}{c}0,124 * * * \\
(0,0128) \\
\end{array}$ & $\begin{array}{c}-0,242^{*} * * \\
(0,0152)\end{array}$ \\
\hline hnos 1 & $\begin{array}{c}0,423 * * * \\
(0,0706)\end{array}$ & $\begin{array}{l}-0,0715 \\
(0,0916)\end{array}$ & $\begin{array}{c}0,260^{* * *} * \\
(0,0117)\end{array}$ & $\begin{array}{c}-0,108 * * * \\
(0,0158)\end{array}$ & $\begin{array}{c}0,237 * * * \\
(0,0121)\end{array}$ & $\begin{array}{c}-0,183^{* * * *} \\
(0,0113)\end{array}$ \\
\hline hnos2 & $\begin{array}{c}0,294^{* * *} * \\
(0,0695)\end{array}$ & $\begin{array}{c}-0,208^{* *} \\
(0,0980)\end{array}$ & $\begin{array}{c}0,246^{* * *} \\
(0,0127)\end{array}$ & $\begin{array}{c}-0,166^{* * *} \\
(0,0180) \\
\end{array}$ & $\begin{array}{c}0,216^{* * * *} \\
(0,0133)\end{array}$ & $\begin{array}{c}-0,158^{* * *} \\
(0,0145) \\
\end{array}$ \\
\hline hnos3 & $\begin{array}{l}0,173 * * \\
(0,0762)\end{array}$ & $\begin{array}{c}0,000835 \\
(0,121) \\
\end{array}$ & $\begin{array}{c}0,127 * * * \\
(0,0137) \\
\end{array}$ & $\begin{array}{c}-0,0612^{* * *} * \\
(0,0231)\end{array}$ & $\begin{array}{c}0,172 * * * \\
(0,0160)\end{array}$ & $\begin{array}{l}-0,0302 \\
(0,0222)\end{array}$ \\
\hline hnos4 & $\begin{array}{c}-0,122 \\
(0,0905) \\
\end{array}$ & $\begin{array}{c}0,166 \\
(0,201) \\
\end{array}$ & $\begin{array}{c}-0,160^{* * * *} \\
(0,0166) \\
\end{array}$ & $\begin{array}{l}0,00357 \\
(0,0379) \\
\end{array}$ & $\begin{array}{c}0,152^{* * * *} \\
(0,0194) \\
\end{array}$ & $\begin{array}{c}0,339 * * * \\
(0,0457) \\
\end{array}$ \\
\hline hijo2 & $\begin{array}{c}-0,223^{* * *} \\
(0,0562) \\
\end{array}$ & $\begin{array}{c}0,241^{* * *} * \\
(0,0716)\end{array}$ & $\begin{array}{c}-0,135^{* * *} \\
(0,00954) \\
\end{array}$ & $\begin{array}{c}0,0979 * * * \\
(0,0120)\end{array}$ & $\begin{array}{c}-0,161^{* * * *} \\
(0,0101) \\
\end{array}$ & $\begin{array}{l}0,148 * * * \\
(0,00990) \\
\end{array}$ \\
\hline hijo3 & $\begin{array}{c}-0,155^{* *} \\
(0,0737)\end{array}$ & $\begin{array}{c}0,304 * * * \\
(0,104)\end{array}$ & $\begin{array}{c}-0,161^{* * * *} \\
(0,0131)\end{array}$ & $\begin{array}{c}0,285^{* * * *} \\
(0,0194)\end{array}$ & $\begin{array}{c}-0,248^{* * * *} \\
(0,0145) \\
\end{array}$ & $\begin{array}{c}0,0838^{* * * *} \\
(0,0181)\end{array}$ \\
\hline hijo4 & $\begin{array}{c}-0,205^{* *} \\
(0,0896)\end{array}$ & $\begin{array}{c}0,135 \\
(0,167) \\
\end{array}$ & $\begin{array}{c}-0,130^{* * * *} \\
(0,0169) \\
\end{array}$ & $\begin{array}{c}0,307 * * * \\
(0,0378)\end{array}$ & $\begin{array}{c}-0,181 * * * \\
(0,0221) \\
\end{array}$ & $\begin{array}{c}-0,123 * * * \\
(0,0339)\end{array}$ \\
\hline pric_padres & $\begin{array}{c}0,0253 \\
(0,0506)\end{array}$ & $\begin{array}{c}0,374^{* * *} \\
(0,0779)\end{array}$ & $\begin{array}{c}-0,0275^{* * *} \\
(0,00970)\end{array}$ & $\begin{array}{c}0,488^{* * * *} \\
(0,0173)\end{array}$ & $\begin{array}{c}-0,0297 * * * \\
(0,0114)\end{array}$ & $\begin{array}{c}0,579 * * * \\
(0,0149)\end{array}$ \\
\hline seci_padres & $\begin{array}{c}0,401 * * * \\
(0,0521) \\
\end{array}$ & $\begin{array}{l}-0,121^{*} \\
(0,0730)\end{array}$ & $\begin{array}{l}0,312^{* * *} * \\
(0,00955) \\
\end{array}$ & $\begin{array}{c}0,386^{* * * *} \\
(0,0143)\end{array}$ & $\begin{array}{c}0,285^{* * * *} \\
(0,0113) \\
\end{array}$ & $\begin{array}{c}0,172 * * * \\
(0,0122) \\
\end{array}$ \\
\hline secc_padres & $\begin{array}{c}0,451 * * \\
(0,227) \\
\end{array}$ & $\begin{array}{r}-0,0641 \\
(0,237) \\
\end{array}$ & $\begin{array}{c}0,555^{* * *} * \\
(0,0155) \\
\end{array}$ & $\begin{array}{l}-0,0211 \\
(0,0136)\end{array}$ & $\begin{array}{c}0,519^{* * * *} \\
(0,0163) \\
\end{array}$ & $\begin{array}{c}-0,248^{* * *} \\
(0,0111) \\
\end{array}$ \\
\hline supi_padres & $\begin{array}{c}1,040 * * * \\
(0,205) \\
\end{array}$ & $\begin{array}{l}-0,225^{* *} \\
(0,0928) \\
\end{array}$ & $\begin{array}{c}0,502^{* * *} * \\
(0,0177)\end{array}$ & $\begin{array}{c}-0,179 * * * \\
(0,0141) \\
\end{array}$ & $\begin{array}{c}0,374 * * * \\
(0,0219) \\
\end{array}$ & $\begin{array}{c}-0,428 * * * \\
(0,0127) \\
\end{array}$ \\
\hline supc_padres & $\begin{array}{c}1,011 * * * \\
(0,159) \\
\end{array}$ & $\begin{array}{c}-0,477 * * * \\
(0,0872) \\
\end{array}$ & $\begin{array}{c}0,584 * * * \\
(0,0243) \\
\end{array}$ & $\begin{array}{c}-0,265^{* * * *} \\
(0,0154) \\
\end{array}$ & $\begin{array}{c}0,339 * * * \\
(0,0203) \\
\end{array}$ & $\begin{array}{c}-0,366^{* * * *} \\
(0,0128)\end{array}$ \\
\hline ocupado_jefe & $\begin{array}{c}-0,114 \\
(0,0762)\end{array}$ & $\begin{array}{r}-0,0591 \\
(0,130)\end{array}$ & $\begin{array}{c}0,0516^{* * * *} \\
(0,0110)\end{array}$ & $\begin{array}{c}0,0673^{* * *} \\
(0,0183)\end{array}$ & $\begin{array}{c}0,183^{* * *} * \\
(0,0116)\end{array}$ & $\begin{array}{l}0,0278^{*} \\
(0,0158)\end{array}$ \\
\hline dos_pea & $\begin{array}{c}0,229 * * * \\
(0,0522)\end{array}$ & $\begin{array}{c}0,265^{* * * *} \\
(0,0679)\end{array}$ & $\begin{array}{l}0,125^{* * * *} \\
(0,00951)\end{array}$ & $\begin{array}{c}-0,0673^{* * *} * \\
(0,0140)\end{array}$ & $\begin{array}{l}0,00385 \\
(0,0123)\end{array}$ & $\begin{array}{c}0,123^{* * *} * \\
(0,0129)\end{array}$ \\
\hline metrop & $\begin{array}{c}0,0366 \\
(0,0484) \\
\end{array}$ & $\begin{array}{c}-0,737 * * * \\
(0,0716) \\
\end{array}$ & $\begin{array}{c}-0,147^{* * *} \\
(0,00822) \\
\end{array}$ & $\begin{array}{c}-0,884^{* * * *} \\
(0,0130)\end{array}$ & $\begin{array}{c}-0,0108 \\
(0,00855) \\
\end{array}$ & $\begin{array}{c}-0,568^{* * *} \\
(0,00890) \\
\end{array}$ \\
\hline ipcf & $\begin{array}{c}7,26 \mathrm{e}-05^{* * * *} * \\
(8,81 \mathrm{e}-06) \\
\end{array}$ & $\begin{array}{c}-0,000113^{* * * *} \\
(6,05 \mathrm{e}-06)\end{array}$ & $\begin{array}{c}0,000114^{* * * *} \\
(2,60 \mathrm{e}-06) \\
\end{array}$ & $\begin{array}{c}-0,000110^{* * * *} \\
(1,15 \mathrm{e}-06) \\
\end{array}$ & $\begin{array}{c}4,22 \mathrm{e}-05^{*} * * \\
(1,44 \mathrm{e}-06) \\
\end{array}$ & $\begin{array}{c}-0,000153^{* * * *} \\
(1,14 \mathrm{e}-06) \\
\end{array}$ \\
\hline Constante & $\begin{array}{c}5,798 * * * \\
(0,242) \\
\end{array}$ & $\begin{array}{c}0,489 \\
(0,305) \\
\end{array}$ & $\begin{array}{c}4,985^{* * * *} \\
(0,0394) \\
\end{array}$ & $\begin{array}{l}1,031 * * * \\
(0,0520)\end{array}$ & $\begin{array}{l}4,966^{* * * *} \\
(0,0445)\end{array}$ & $\begin{array}{c}0,703 * * * \\
(0,0418) \\
\end{array}$ \\
\hline Obs & 6,1 & 3,781 & 233,445 & 156,648 & 282,726 & 212,289 \\
\hline
\end{tabular}

Nota: Estimaciones Probit. Individuos entre 12 a 17 años que asisten a educación secundaria para el total del país.

Fuente: Elaboración propia en base a datos de ECH. 


\section{Breve CV de los autores}

\section{Lucía Ramírez Leira}

Investigadora del Centro de Estudios Distributivos, Laborales y Sociales (CEDLAS) de la Universidad Nacional de La Plata (UNLP); e investigadora del Instituto de Economía (IECON) de la Universidad de la República (UDELAR). Ramírez es Licenciada en Economía de la Universidad de la República (Uruguay); Magíster en Economía de la Universidad Nacional de La Plata.; y candidata a doctora de la UNLP. Es docente de grado de la Licenciatura en Economía de la UNLP; y de posgrado en la Maestría en Economía (UNLP). Sus áreas de investigación son economía de la educación, economía laboral y desigualdad, entre otras. ORCID ID: https://orcid.org/0000-0001-9747-5106. Email: lucia.ramirez@econo.unlp.edu.ar

\section{Emmanuel Vazquez}

Investigador del Centro de Estudios Distributivos, Laborales y Sociales (CEDLAS) de la Universidad Nacional de La Plata (UNLP). Vazquez es Licenciado en Economía de la Universidad Nacional de La Plata y Magíster en Economía de la Universidad de California, Los Ángeles (UCLA). Actualmente es profesor de grado y posgrado en la UNLP e investigador categorizado de esta institución. Sus investigaciones sobre temas de educación y mercados laborales han sido publicadas en revistas académicas tanto nacionales como internacionales. Ha sido consultor de numerosas instituciones tales como el Banco Mundial, la Comisión Económica para América Latina y el Caribe (CEPAL), la Corporación Andina de Fomento (CAF) y el Banco Interamericano de Desarrollo (BID). Vazquez ha obtenido varios premios, incluyendo distinciones de la UNLP a sus mejores egresados de grado y posgrado, y de la Asociación Argentina de Economía Política (AAEP) en el contexto del Premio Investigador Joven. ORCID ID: https://orcid.org/0000-0003-3061-6618. Email: evazquez@cedlas.org 